\title{
ADA for IBL: Lessons Learned in Aligning Learning Design and Analytics for Inquiry-Based Learning Orchestration
}

\author{
María Jesús Rodríguez-Triana ${ }^{1}$, Luis P. Prieto ${ }^{2}$, Yannis Dimitriadis ${ }^{3}$, Ton de Jong ${ }^{4}$, Denis Gillet ${ }^{5}$
}

\begin{abstract}
Orchestrating technology-enhanced learning is a difficult task, especially in demanding pedagogical approaches like inquiry-based learning (IBL). To foster effective teacher adoption, both the complexity of designing IBL activities and the uncertainty about the student learning path during enactment need to be addressed. Previous research suggests that aligning learning design and learning analytics can be an effective way to provide such orchestration support. This paper reports on a design-based research (DBR) project to explore teachers' orchestration needs in Go-Lab (a technological ecosystem for IBL used by thousands of primary and secondary school teachers around the world), and on how solutions that align learning design and analytics can fulfill such needs. The analysis of data from multiple events (including surveys, case studies, workshops with teachers, and platform usage analyses) led to a catalogue of IBL orchestration needs that can be tackled by aligning learning design and analytics, as well as a list of guidelines for technology development aiming to support IBL orchestration. These two contributions can support the creation of future learning analytics-enhanced IBL environments that are both pedagogically grounded and usable by teachers in authentic settings.
\end{abstract}

\section{Notes for Practice}

- Inquiry-based learning (IBL) has many documented benefits, but its demanding orchestration hinders adoption by teachers.

- We identified teachers' orchestration needs in an IBL platform and explored how to fulfill those needs with solutions that align learning design and learning analytics.

- Researchers, designers, and developers of information and communications technology (ICT) for IBL orchestration can benefit from the elicited IBL orchestration needs and design guidelines reported.

\section{Keywords}

Learning design, learning analytics, inquiry-based learning, orchestration, technology-enhanced learning

Submitted: 20/09/20 — Accepted: 13/07/21 — Published: 03/09/21

Corresponding author ${ }^{1}$ Email: mjrt@tlu.ee Address: School of Digital Technologies, Tallinn University, Narva rd 25, 10120 Tallinn, Estonia. ORCID ID: https://orcid.org/0000-0001-8639-1257

${ }^{2}$ Email: Iprisan@tlu.ee Address: School of Educational Sciences, Tallinn University, Narva rd 25, 10120 Tallinn, Estonia. ORCID ID: https://orcid.org/0000-0002-0057-0682

3Email: yannis@tel.uva.es Address: School of Telecommunications Engineering, Universidad de Valladolid, Paseo de Belén 15, 47011 Valladolid, Spain. ORCID ID: https://orcid.org/0000-0001-7275-2242

${ }^{4}$ Email: a.j.m.dejong@utwente.nl Address: Faculty of Behavioral, Management and Social Sciences, University of Twente, Cubicus B228, 7500-AE Enschede, Netherlands. ORCID ID: http:// orcid.org/0000-0003-0416-4304

${ }^{5}$ Email: denis.gillet@epfl.ch Address: School of Engineering, École Polytechnique Fédérale de Lausanne, Station 11, CH-1015 Lausanne, Switzerland. ORCID ID: https://orcid.org/0000-0002-2570-929X

\section{Introduction}

Inquiry-based learning (IBL) is an educational strategy in which students actively construct knowledge following the methods and practices of scientists. This demands that students develop their questioning skills, make hypotheses, design experiments, and reflect on the resulting observations. Although this pedagogical approach has many benefits (e.g., improved ability to apply 
scientific thinking, preparedness for graduate study, increased retention rates) (Seymour et al., 2004), the literature also shows that IBL depends on the provision of adequate scaffolds (Alfieri et al., 2011; Furtak et al., 2012; Zacharia et al., 2015), making it notoriously demanding for teachers (de Jong et al., 2013; Dobber et al., 2017; Kirschner et al., 2006). In IBL, teachers need to strike a balance between letting students freely explore on the one hand and guiding them to avoid frustration, focusing their attention on the target phenomenon, and making the inquiry feasible in the allotted time on the other. Teachers also need to carefully choose motivating topics, narrow down and describe the scope of student inquiries, and guide the IBL process, monitoring each individual's learning path to intervene in case of need. Such overload makes the large-scale adoption of IBL a challenging matter (Maaß \& Artigue, 2013).

There have been several attempts in the technology-enhanced learning (TEL) research community to support teachers in the orchestration of IBL, i.e., the productive coordination of multiple learning activities occurring at different social levels, across contexts and media (Dillenbourg \& Jermann, 2010; Prieto et al., 2011). To address the complexity of designing effective inquiries, the field of learning design has provided guidelines and best practices to help teachers structure the sequence of inquiry tasks, resources, and scaffolds for students throughout the IBL process (Sharples \& Anastopoulou, 2012). Also, the field of learning analytics has proposed solutions that collect, analyze, and report data about learners and their contexts to support IBL orchestration, e.g., by enabling awareness and reflection on students' activities and progress (Manske et al., 2014; Vozniuk et al., 2015).

Despite these efforts, the design of IBL tools suitable for teachers' everyday practice remains a "grand challenge" in TEL (Pedaste, Lazonder, et al., 2015). Orchestration research posits that, to favour adoption, technologies and instructional designs need to take into account the pragmatic constraints and needs of actual classrooms (Dillenbourg \& Jermann, 2010), and considers that their support needs to be aligned, not looked at in isolation (Prieto et al., 2011). Similar calls for alignment have been made by researchers in both the learning design and learning analytics fields to provide more meaningful and contextualized analytics, as well as informed and assessed learning designs (Lockyer et al., 2013). Examples of such "alignment of design and analytics" (ADA from now on) have been proposed in IBL itself (Guéraud et al., 2009; Sergis et al., 2019). Yet, due to the scarcity of (explicit, formal) learning designs that fit everyday educational practice, the implementation of these ADA approaches remains largely theoretical or small scale.

In this paper, we explore such ADA support and how it can help teachers orchestrate (technology-enhanced) IBL ${ }^{1}$. Concretely, our research questions are as follows: (RQa) "What are the IBL orchestration needs of teachers?" and (RQb) "To what extent do ADA solutions fulfill those needs?" We tackled these questions in a four-year, iterative design-based research (DBR) process (Wang \& Hannafin, 2005) in the context of Go-Lab (a TEL initiative to promote and support IBL in primary and secondary education). The three DBR iterations presented in this paper span one survey study, eight case studies of IBL implementation in authentic classrooms, four teacher workshops, and an analysis of the usage of the Go-Lab authoring platform. All in all, 95 different STEM teachers were actively involved. In these studies, multiple data-gathering techniques (questionnaires, interviews, observations, focus groups, log data gathering) and mixed methods of analysis were used.

\section{Related Work}

\subsection{Orchestrating IBL}

The lack of adoption of IBL in our classrooms can be related to personal beliefs and predispositions (Silm et al., 2017; Voet \& De Wever, 2019) or external factors (e.g., dissemination of IBL initiatives, professional development, or resource availability) (Dorier \& García, 2013; Hofer \& Lembens, 2019; Maaß \& Artigue, 2013). It can also be due to the considerable effort and changes in teaching practices required to effectively guide IBL (Harris \& Rooks, 2010). In this paper, we focus specifically on how technology can support teachers in the design and enactment of inquiry activities in authentic classroom conditions - what Dillenbourg \& Jermann (2010) would call the "orchestration" of IBL.

In their literature review, Prieto and colleagues (2011) proposed a conceptual framework that decomposes the orchestration of TEL into several aspects that need to be supported by technology: the design of learning activities, as well as their enactment (i.e., the regulation and management of activities; their awareness, assessment, and reflection; and their adaptation under unexpected occurrences). This framework also includes other factors, like the role of the teacher and other actors in the orchestration, their theories and beliefs, the pragmatic constraints of the setting, and the alignment of all these elements to produce an effective learning experience. Hence, to develop IBL technologies that are easily adoptable, we need to understand the main challenges that teachers face to effectively orchestrate IBL (so that technology can address those needs).

Among the aforementioned orchestration support aspects, the most commonly addressed in the IBL literature is the design of inquiries. This includes the ability to author scripts of IBL activities (Lejeune et al., 2009; Mulholland et al., 2012; Slotta et al., 2013) and the modification of pre-made scripts to address the level and skills of students (Friesel et al., 2015; Gillet

\footnotetext{
${ }^{1}$ In the remainder of the paper, we will focus on technology-enhanced IBL, but we will refer to it simply as "IBL," for brevity and readability. 
et al., 2013). Another commonly supported IBL orchestration need is awareness, which enables teachers to monitor students' progress (Mulholland et al., 2012; Slotta et al., 2013) and provide them with feedback (Jona \& Uttal, 2013). Examples can also be found of support for run-time management of the learning activities (Lejeune et al., 2009; Slotta et al., 2013) or the dynamic adaptation of planned scripts (Mulholland et al., 2012).

More specific to IBL proposals and pedagogy is the emphasis on subverting the traditional role of the teacher as the main orchestrator of activities, instead enabling "personal inquiries" (Mulholland et al., 2012) or off-loading orchestration tasks from the teacher to allow student self-orchestration (Blake et al., 2013; Dobber et al., 2017; Sharples, 2013). Other IBL-specific needs include the pragmatic adaptation of the inquiry design to the contextual constraints of the classroom (Friesel et al., 2015), e.g., enabling offline usage in inquiries that take students far from reliable network infrastructures (Mulholland et al., 2012). Many IBL proposals also provide coarse-grained support for the enactment of the inquiry itself, in the form of spaces for different phases of the inquiry cycle (Gillet et al., 2013; Mulholland et al., 2012).

\subsection{Aligning (Learning) Design and Analytics}

The orchestration aspect that has received the least attention so far within IBL research is the alignment of the different supports that teachers are offered. For instance, a technology may provide conceptual support on how to design effective inquiries (Hsu et al., 2015; Pedaste, Mäeots, et al., 2015), while learning analytics can help monitor learner progress (e.g., through widgets (Manske et al., 2014)). However, those aids are usually provided independent of each other. Teachers and learners are left to bridge the gap between their particular inquiry design and the data provided by the analytics solutions, thus limiting their interpretability and actionability due to a lack of context (Reimann, 2016). Besides, practitioners cannot easily monitor or assess the effectiveness of IBL designs, since learning analytics solutions normally do not analyze the data from a learning design perspective (Sergis et al., 2019).

Researchers have advocated for the alignment between learning design and analytics (ADA) in TEL (Bakharia et al., 2016; Lockyer et al., 2013). This alignment may bring benefits such as the use of learning designs to better understand and interpret analytics in its pedagogical context (Lockyer \& Dawson, 2011; Shen et al., 2020) (e.g., using student information set at design time). Conversely, learning analytics could help improve a pedagogical design (Emin-Martinez et al., 2014; Hernández-Leo et al., 2018; Mangaroska \& Giannakos, 2018), e.g., through detecting deviations from the original plan or identifying aspects that may require revision based on metrics obtained while enacting the design (Emin-Martinez et al., 2014; Lockyer et al., 2013).

Examples exist of ADA implementations in authentic classroom settings at different granularity levels. At the activity level, teachers can be informed about students reaching the goals of a simulation-based learning activity, as well as errors detected (Guéraud et al., 2009). At the lesson level, deviations between expected and actual student interactions have been reported during a collaborative lesson (Rodríguez-Triana et al., 2015). At the course level, analytics can relate types of learning activities in a course design to learners' behaviour and performance (Rienties et al., 2015). At the curriculum level, analytics can help compare course specifications and actual student performance (Gluga et al., 2013). Yet, at the granularity levels that orchestration research considers (activity/lesson), ADA proposals remain largely theoretical and/or small scale (Mangaroska \& Giannakos, 2018). Indeed, there are still few examples of ADA implementations in IBL (Wiley et al., 2020). This may be related to the lack of explicit, machine-readable learning designs in authentic educational practice. Thus, to align learning design and analytics in inquiry settings, we need TEL solutions that support teachers across all orchestration activities, from design creation to deployment and enactment with students, along with collection of traces during this enactment (Sergis et al., 2019).

This overview of TEL and IBL research highlights the importance of supporting teachers in different IBL orchestration tasks (as per Prieto and colleagues', 2011, orchestration framework), as well as the fact that solutions aligning learning design and analytics can be appropriate to provide such support in a coherent, pedagogically grounded manner. This was the starting point for our DBR into whether and how ADA solutions can effectively support teachers' IBL orchestration needs. This DBR is described in the following sections.

\section{Context and Methodology}

This paper describes a four-year research effort guided by two questions: "What are the orchestration needs of teachers implementing IBL in their classrooms?" (RQa) and "To what extent do ADA solutions fulfill such orchestration needs?" $(\mathrm{RQb})$. These questions were explored in the context of a particular initiative to support IBL in primary and secondary schools (Go-Lab), although we hope its implications and lessons learned are relevant for researchers and technology designers aiming to support IBL using other platforms. 


\subsection{Research Context: Go-Lab}

The Go-Lab ${ }^{2}$ initiative provides an ecosystem of tools that supports IBL and several related orchestration tasks, including co-design and enactment support via inquiry learning spaces (ILSs) (de Jong et al., 2021). ILSs are by default structured according to the different phases of an inquiry process (orientation, conceptualization, investigation, conclusion, and discussion; see Pedaste, Mäeots, et al., 2015). Teachers can later modify this structure depending on their learning goals (see the left side of Figure 1). Each of these phases provides students with background information and tasks to perform (e.g., proposing hypotheses, collecting observations of a phenomenon, interacting with a simulation). Teachers can enrich these phases with additional resources, such as multimedia content, scaffolding apps (e.g., supporting hypothesis formulation), and labs. In an ILS (representing an inquiry), learners can thus navigate through different inquiry phases, performing associated tasks and interacting with various multimedia resources to accomplish them.

To help teachers in ILS design, Go-Lab provides a repository ${ }^{3}$ where teachers can find and reuse online labs, applications (or apps), and existing ILSs. Go-Lab also offers Graasp ${ }^{4}$, the ILS orchestration platform where teachers can (co)create ILSs (from scratch or by adapting existing ones), share them with students, and follow students' progress.

By 2018, more than 27,000 primary and secondary school teachers worldwide had signed up in Graasp, out of which around 16,000 actively (co)created ILSs during 2015-2018 (the four-year period reported in this paper). In the research below, this large community of practitioners helped us understand the orchestration needs when implementing IBL in their classrooms (RQa), and to what extent ADA solutions could fulfill such orchestration needs (RQb).

\subsection{Methodology}

To explore the aforementioned research questions, we followed DBR (Wang \& Hannafin, 2005), a research methodology often used to study educational environments through iterative cycles of design, implementation, and analysis. Following DBR guidelines (Barab \& Squire, 2004; Wang \& Hannafin, 2005), this research is based on collaboration between researchers and practitioners in real-world settings, which led us to the contextually sensitive (in the sense of "fit for the studied contexts") findings and design guidelines presented in Section 7. Since DBR may not be replicated and generalized in the classical sense, we adopted the following measures to provide research outcomes that can eventually be adapted to different contexts. First, we provide a detailed description of the design and the context where each study took place to help readers understand the extent to which our results and guidelines could apply to their own contexts. Second, instead of paying attention to context-specific problems, we attempt to address systemic issues that arise in many real-world settings (i.e., persistent problems of practice around IBL orchestration). And third, to conduct research in a real-world setting that could be representative of many others, we chose a technological ecosystem for IBL used by thousands of primary and secondary school teachers around the world. Also, our studies involved teachers with different levels of expertise, from different STEM subjects and countries. Moreover, this research employs mixed methods, typically used in DBR to enable the exploration of the different perspectives and multiple factors that affect learning situations.

Our DBR comprised three iterations combining explorative purposes (to identify teachers' orchestration needs in IBL, RQa) and evaluative ones (to assess different ADA solutions that try to address such needs, RQb). Each iteration was composed of several data-gathering events (Figure 2):

1. The first iteration was purely exploratory. We conducted a survey study with 21 teachers (experts in IBL and Go-Lab) to better understand their learning contexts and identify perceived information needs when orchestrating ILSs (Event_1).

2. Based on the needs elicited in the first iteration, a second iteration aimed at (a) uncovering further teacher needs emerging while actually orchestrating IBL (using Go-Lab's technologies) in their classrooms, (b) prioritizing the list of needs, and (c) evaluating the suitability of initial ADA solutions for such needs (see the tools evaluated in Events 2 to 4 in Table 2). This iteration involved eight in-depth case studies of IBL enactments in real classrooms, with three different teachers (Event_2) and two face-to-face workshops, one with 28 expert Go-Lab teachers (Event_3) and another with 6 pre-service teachers (Event_4).

3. In the third iteration, we refined the initial ADA solutions and assessed to what extent the solutions (see the tools evaluated in Events 5 to 7 in Table 2) fulfilled the orchestration needs elicited originally. We also identified emergent challenges for IBL adoption. In this iteration, two workshops took place (Event_5 and Event_6), with 34 and 22 teachers (experts in Go-Lab and IBL/ Go-Lab, respectively). Additionally, we analyzed the actual adoption of the ADA solutions among the 15,894 teachers who created ILSs during this four-year period (Event_7).

\footnotetext{
${ }^{2}$ Go-Lab: https://www.golabz.eu

${ }^{3}$ Go-Lab repository: https://www.golabz.eu

${ }^{4} \mathrm{Go}-$ Lab orchestration platform: https://graasp.eu
} 


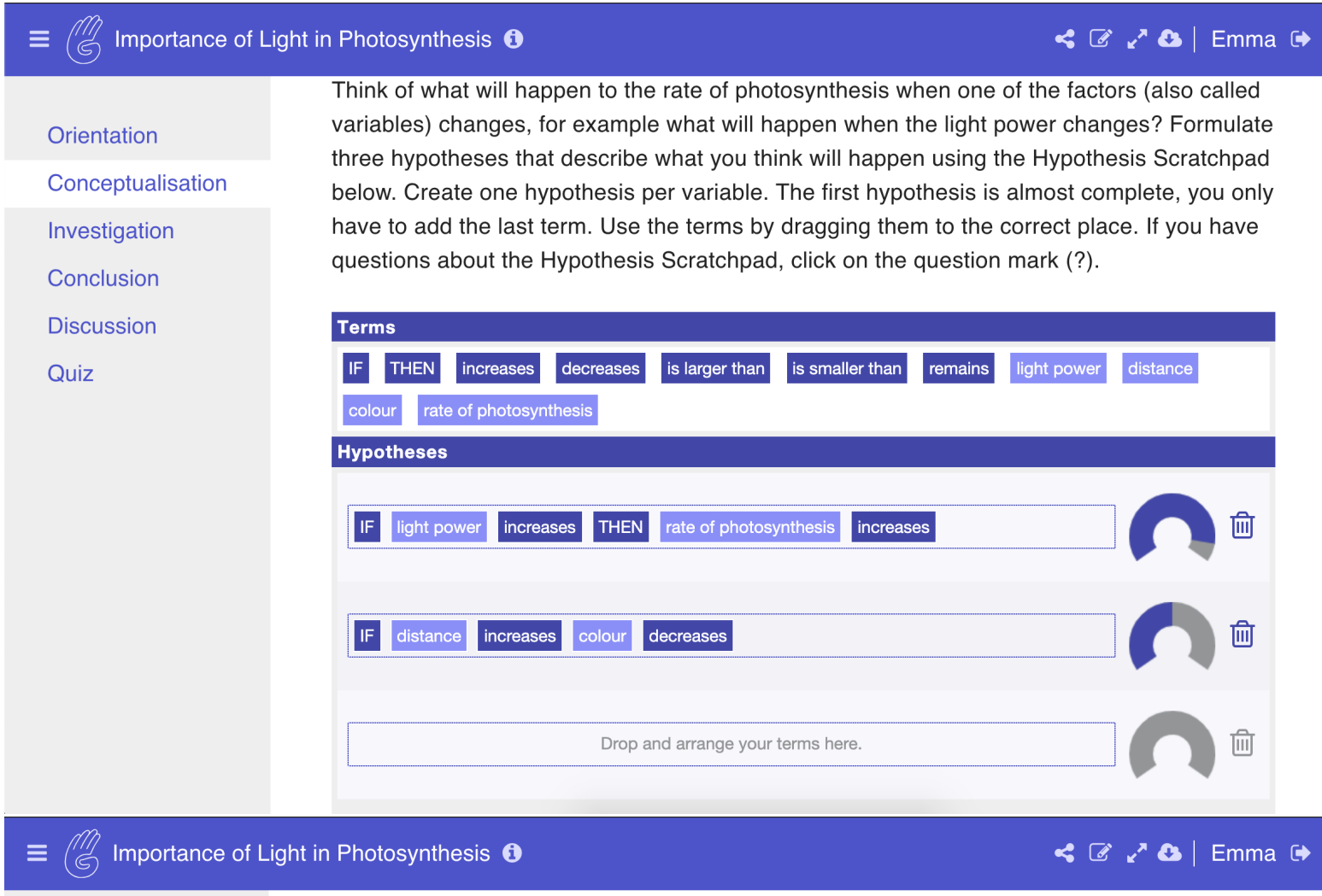

Orientation

Show Procedure

Conceptualisation

Investigation

Conclusion

Discussion

Quiz

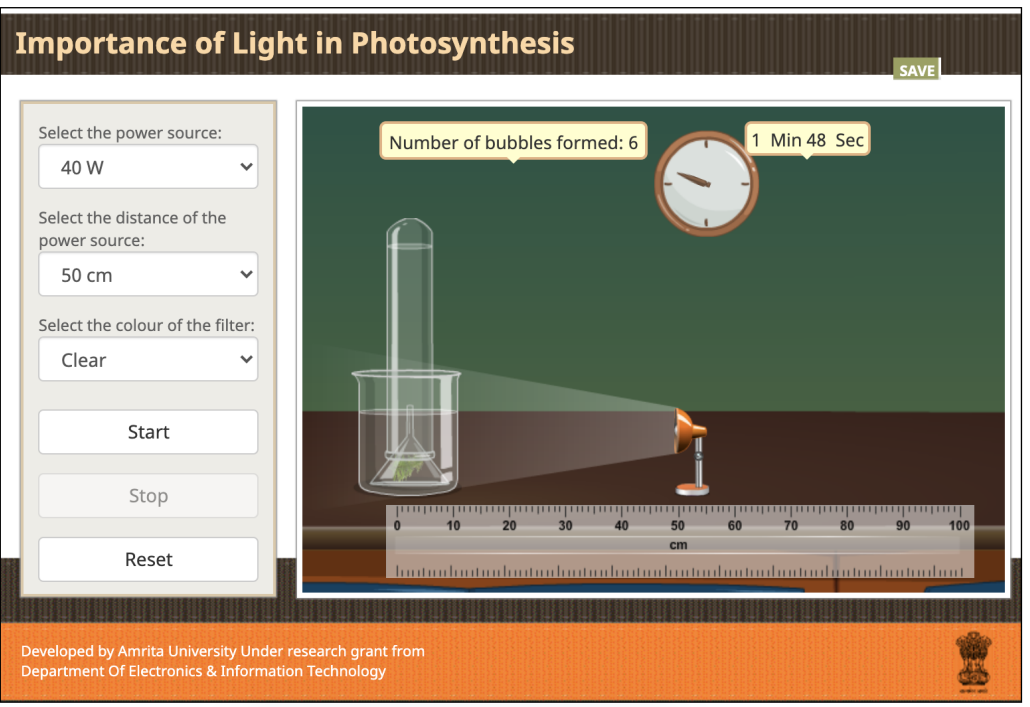

Figure 1. ILS example. Left side: tabs named after inquiry phases (orientation, conceptualization, investigation, conclusion, and discussion), plus an additional teacher-created phase with a quiz. Right side: contents of two of the phases: a scaffolding app (Hypothesis Scratchpad, top) and a virtual laboratory (Amrita University's photosynthesis lab, bottom).

As a result of these iterations, we obtained a final list of teacher orchestration needs and a set of guidelines for the design of ADA solutions for IBL (see the "Discussion" section at the end of this paper). Figure 5 summarizes the main outcomes and decisions made per iteration. In all the aforementioned events, explicit informed consent was obtained from participants after details of the research project were described by the researchers. Additionally, the data analyses of Event_7 are compliant with 


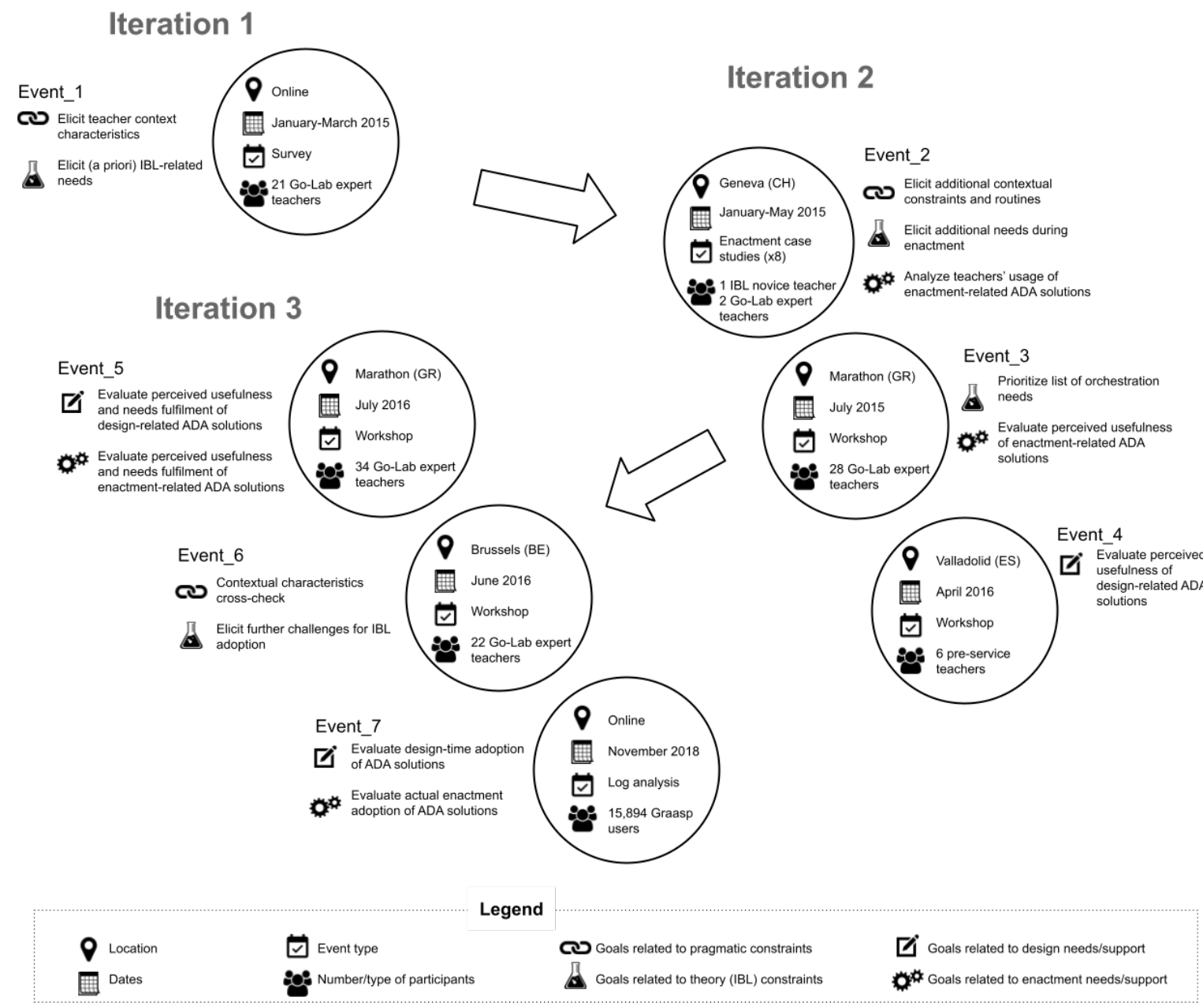

Figure 2. Overview of data-gathering events and their goals, in our DBR iterations.

Graasp's user agreement on data management.

In order to guide our mixed-methods analysis of these events and to gain a holistic understanding of the orchestration of IBL in the different settings studied, we used the orchestration aspects of the conceptual framework proposed by Prieto and colleagues (2011). Following the example and recommendations of other researchers who have applied this framework (Prieto, 2015), we have clustered these aspects into three different areas (see the clouds in Figure 3): looking at the different activities that (IBL) orchestration entails, at the actors that perform these activities, and at the background that shapes the way orchestration is performed. In terms of activities, we will focus on the needs and ADA support for the design and enactment activities. Regarding the background, this study deals with pragmatic and theoretical constraints derived from the pedagogical approach (i.e., specific to $I B L$ ). Finally, given that in all our data-gathering events the teacher was the main driver of the inquiries, we chose not to analyze this aspect specifically (i.e., it was an invariant in our studies). The same applies to the alignment aspect, which is implicit in the ADA solutions developed throughout the studies. Since each event followed slightly different approaches, the analyses performed have been described per event in the following sections.

\section{DBR Iteration 1}

The first iteration mainly focused on identifying the orchestration needs of teachers implementing IBL in their classrooms (RQa) (see Figure 5). Although the literature reviewed above provided initial ideas of teachers' orchestration needs (as reported by researchers), we aimed to better understand teachers' own perceived information needs, specifically regarding Go-Lab's ILS support to IBL orchestration. In this and following sections, results are presented per event, followed by overall conclusions per iteration. 


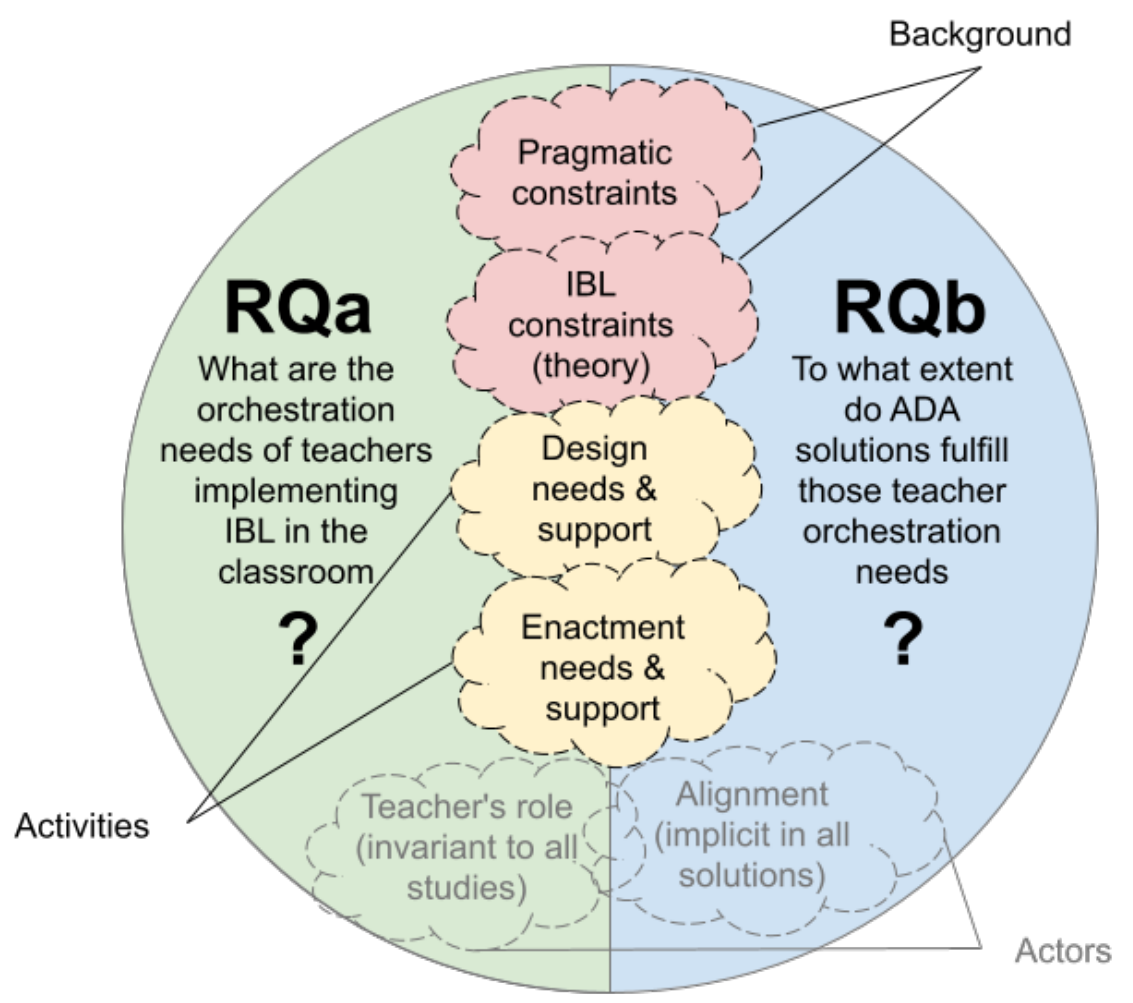

Figure 3. Overall analytical framework of our DBR: research questions and orchestration aspects explored.

\subsection{Event_1 (Online Survey)}

We conducted an online survey ${ }^{5}$ from January to March 2015, aimed at expert teachers. The survey was sent to a group of 21 teachers recruited by the project's national coordinators in nine countries, who looked for teachers with previous experience in Go-Lab motivated to enroll in participatory design activities. The survey aims were (a) to better understand the pragmatic constraints of teachers' classrooms and (b) to identify information needs they had when orchestrating IBL in such contexts (crucial for solutions that align learning design and analytics). Content analysis was applied to the open-ended questions to extract and group teacher needs. We used descriptive statistics to overview teacher needs and constraints.

Regarding teachers' contextual constraints, we posed questions related to three aspects that may add complexity to the orchestration load: the number of students, the delivery mode (inside/outside the classroom), and the social level (e.g., individually, in small groups, etc.) at which the inquiries were conducted in their classroom. Since IBL requires teachers to follow each individual learning path (Dobber et al., 2017; Kirschner et al., 2006; Zacharia et al., 2015), the greater the number of students, the more learning paths have to be followed simultaneously. The physical space where the learning takes place (inside/outside the classroom) also conditions teachers' visual awareness about the learning process (Rodríguez-Triana et al., 2017). Finally, collaborative activities, while highly beneficial, especially for IBL (Pedaste, Mäeots, et al., 2015), may be highly demanding in terms of teacher orchestration (Alavi \& Dillenbourg, 2012; Holstein et al., 2019). The number of students per classroom varied from 12 to 30 . As we can see in Figure 4, teachers used their ILSs totally (47.62\%) or mainly (42.86\%) within the classroom (instead of as homework), with students working either individually or in small groups of two or three students.

In the survey, teachers were asked what information could help them orchestrate ILSs (essential for implementing ADA solutions) through an open question. We classified teachers' expressed needs into 19 clusters (see Table 1, first column) related to the four aspects of our analysis (pragmatic constraints, IBL-related, design-related, and enactment-related). As shown in the column on orchestration aspects, teachers barely reported needs related to IBL specifically, or about their pragmatic constraints, and only two needs related to the design process. The main a priori teacher concerns referred to the enactment (regulation and management, awareness and assessment, adaptation and intervention), with special emphasis on accessing the artifacts generated by students during the inquiry. From a learning design granularity perspective, we can organize these needs into three levels: (a) related to specific resources or tasks included within a phase, (b) focused on the phases of the inquiries' design, and

${ }^{5}$ Event_1 survey: https://goo.gl/KwqVqd 

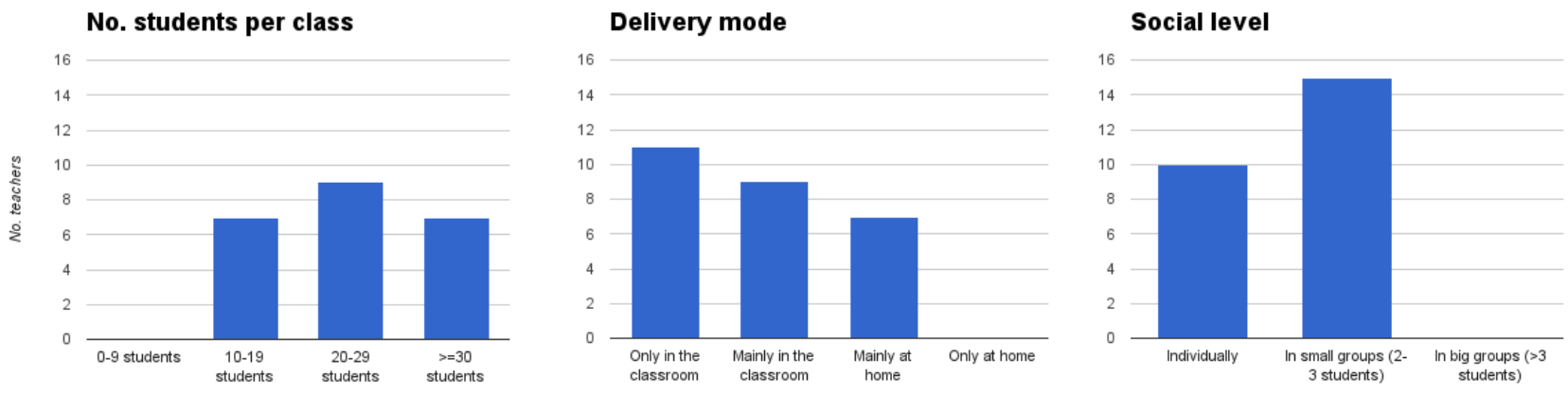

Figure 4. Pragmatic constraints voiced by teachers in the online survey (Event_1).

(c) affecting the whole IBL process.

Table 1. Teacher orchestration needs elicited from a survey (Event_1), later ranked and grouped by importance during a teacher workshop (Event_3). The last column indicates the average and deviation of teachers stating that the orchestration need was satisfied $(-1=$ no, $0=$ partially, $1=$ yes) with the first ADA prototypes developed for the second DBR iteration.

\begin{tabular}{|c|c|c|c|c|c|c|}
\hline \multicolumn{4}{|c|}{ Event_1 (Survey, $n=21)$} & \multicolumn{3}{|c|}{ Event_3 (Workshop, $n=28)$} \\
\hline Teacher Information Needs & $\begin{array}{c}\text { No. } \\
\text { Teacher } \\
\text { Mentions }\end{array}$ & $\begin{array}{c}\text { Orchestration } \\
\text { Aspect }\end{array}$ & $\begin{array}{c}\text { Design } \\
\text { Granularity }\end{array}$ & $\begin{array}{l}\text { Importance } \\
\qquad(\bar{x})\end{array}$ & $\begin{array}{l}\text { Ranking } \\
(\bar{x})\end{array}$ & $\begin{array}{c}\text { Satisfied by First } \\
\text { Prototypes } \\
{[-1,1](\bar{x}, \mathrm{sd})}\end{array}$ \\
\hline Students' questions/comments & 2 & Enactment & Task/resource & High & 1 & $0.42,0.49$ \\
\hline Specifications and tips from other teachers & 1 & IBL, design & Inquiry & High & 2 & $0.33,0.52$ \\
\hline Used resources, apps, labs & 1 & Enactment & Task/resource & High & 2 & $0.79,0.27$ \\
\hline Learning artifacts created by students & 12 & Enactment & Inquiry & High & 3 & $0.33,0.52$ \\
\hline Self-evaluation & 2 & Enactment & Inquiry & High & 4 & $0.67,0.41$ \\
\hline Expert feedback on the ILS design & 1 & IBL, design & Inquiry & Medium & 5 & $0.17,0.41$ \\
\hline Peer evaluation & 1 & Enactment & Inquiry & Medium & 6 & $0.08,0.20$ \\
\hline Stuck students & 2 & Enactment & Inquiry & Medium & 7 & $0.17,0.41$ \\
\hline Followed path & 3 & Enactment & Inquiry & Medium & 8 & $0.67,0.52$ \\
\hline Students who required hints & 1 & Enactment & Task/resource & Medium & 9 & $0.25,0.42$ \\
\hline Statistics per session & 1 & Enactment & Inquiry & Medium & 10 & $0.83,0.41$ \\
\hline Intermediate learning artifacts & 2 & Enactment & Inquiry & Medium & 11 & $0.17,0.41$ \\
\hline Current phase per student/students per phase & 2 & Enactment & Phase & Medium & 12 & $0.67,0.52$ \\
\hline Evidence of face-to-face interaction & 1 & Pragmatic & Task/resource & Medium & 13 & $0.00,0.00$ \\
\hline Current actions & 1 & Enactment & Task/resource & Medium & 14 & $0.67,0.52$ \\
\hline Automatic evaluation & 5 & Enactment & Inquiry & Medium & 15 & $0.17,0.26$ \\
\hline Time spent (per phase, app, ILS) & 4 & Enactment & $\begin{array}{l}\text { Inquiry, phase, } \\
\text { task/resource }\end{array}$ & Medium & 15 & $1.00,0.00$ \\
\hline Current state & 1 & Enactment & Inquiry & Low & 16 & $1.00,0.00$ \\
\hline Visited phases & - & Enactment & Phase & Low & 17 & $0.83,0.41$ \\
\hline
\end{tabular}

\subsection{Iteration 1 Summary}

The main outcomes and next steps of each iteration are represented in Figure 5. In the case of Iteration 1, outcomes were related to eliciting IBL orchestration needs (RQa) according to expert teachers. Still, to better understand how pragmatic constraints (such as the contextual constraints and teachers' classroom management style) influenced these voiced needs, in-depth case studies of IBL enactments in authentic settings were planned for the second iteration. Since the frequency of mentions of a need may not indicate its importance, we would involve teachers actively in the prioritization of needs in a later workshop. In 
addition, the next iteration would also pay attention to the additional requirements of teachers not familiar with IBL/Go-Lab, in order to facilitate their adoption. Furthermore, to explore to what extent ADA solutions could fulfill those needs (RQb), Go-Lab started building a set of ADA solution prototypes to be integrated in the ILSs. These solutions (described in the next section) aimed mainly at teacher enactment activities (especially targeting awareness, assessment, and reflection), which were most frequently mentioned in Iteration 1 .

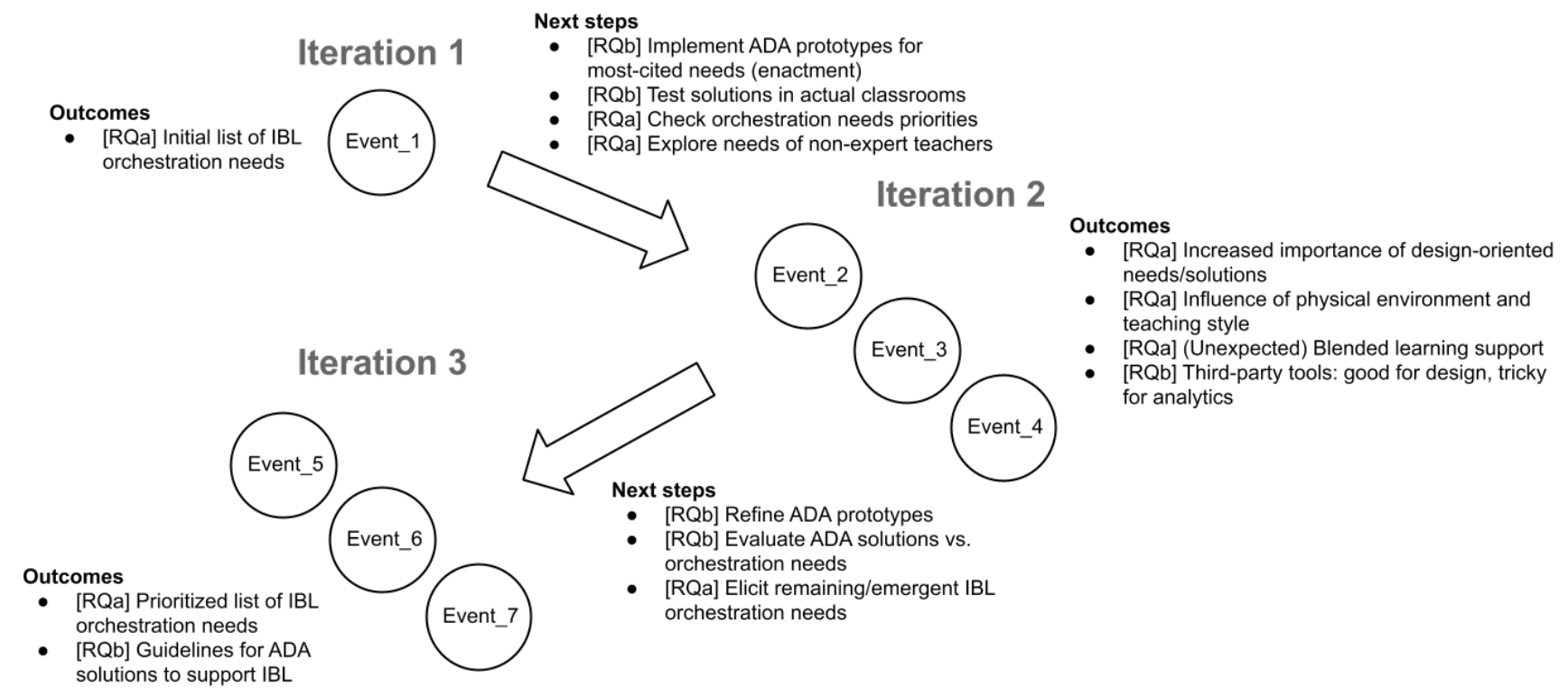

Figure 5. Graphical summary of the main outcomes and next steps prompted by the iterations of our DBR, and their relation to the research questions.

\section{DBR Iteration 2}

This iteration had both an exploratory purpose (to further elicit and prioritize teachers' orchestration needs, RQa) and an evaluative one (assessing a first set of ADA prototypes, RQb). Since teacher expertise might influence their needs, we gathered data from both novice (in fact, pre-service) teachers and practitioners familiar with IBL and Go-Lab.

Based on the first iteration results, a number of ADA solutions were progressively proposed, implemented, and evaluated in Go-Lab (see Table 2 and Figures 6, 8, and 9). We initially focused on enactment-oriented solutions (because those were the most commonly voiced needs) and later on developed design-oriented solutions. Further details about these apps are available in Manske \& Cao (2015) and Rodríguez-Triana \& Holzer (2016), and currently existing ADA solutions can be found in the Go-Lab repository ${ }^{6}$. It is worth noting that certain orchestration needs mentioned by teachers were not addressed exclusively with ADA solutions (see last row in Table 2): additional functionalities were developed to enable access to student artifacts (during and after the activities), collaboration and discussions with other teachers and experts while designing an ILS, synchronous online communication with students, self-evaluation, and peer review, as well as to visualize ILS activity logs. However, the need for evidence of face-to-face interactions or student hint requests was not implemented due to the lack of data within the platform in those regards.

\subsection{Event_2 (Enactment Case Studies)}

To identify further orchestration needs that might only emerge during the actual orchestration of an inquiry using ILSs, and to assess the first round of ADA solutions in authentic practice, we studied eight cases of authentic classroom enactments of IBL using Go-Lab (featuring the first set of ADA solutions). Three teachers (let us call them Alice, Bob, and Chris) enacted a total of eight learning designs with a grand total of 111 students. While Alice had no previous experience in using ILSs or IBL, Bob and Chris had already used ILSs in their courses.

The main data sources used during these eight case studies were teacher interviews and classroom observations. Before the orchestration started, teachers were interviewed about the perceived usefulness of five potential ADA solutions for awareness,

\footnotetext{
${ }^{6}$ https://www.golabz.eu/apps 


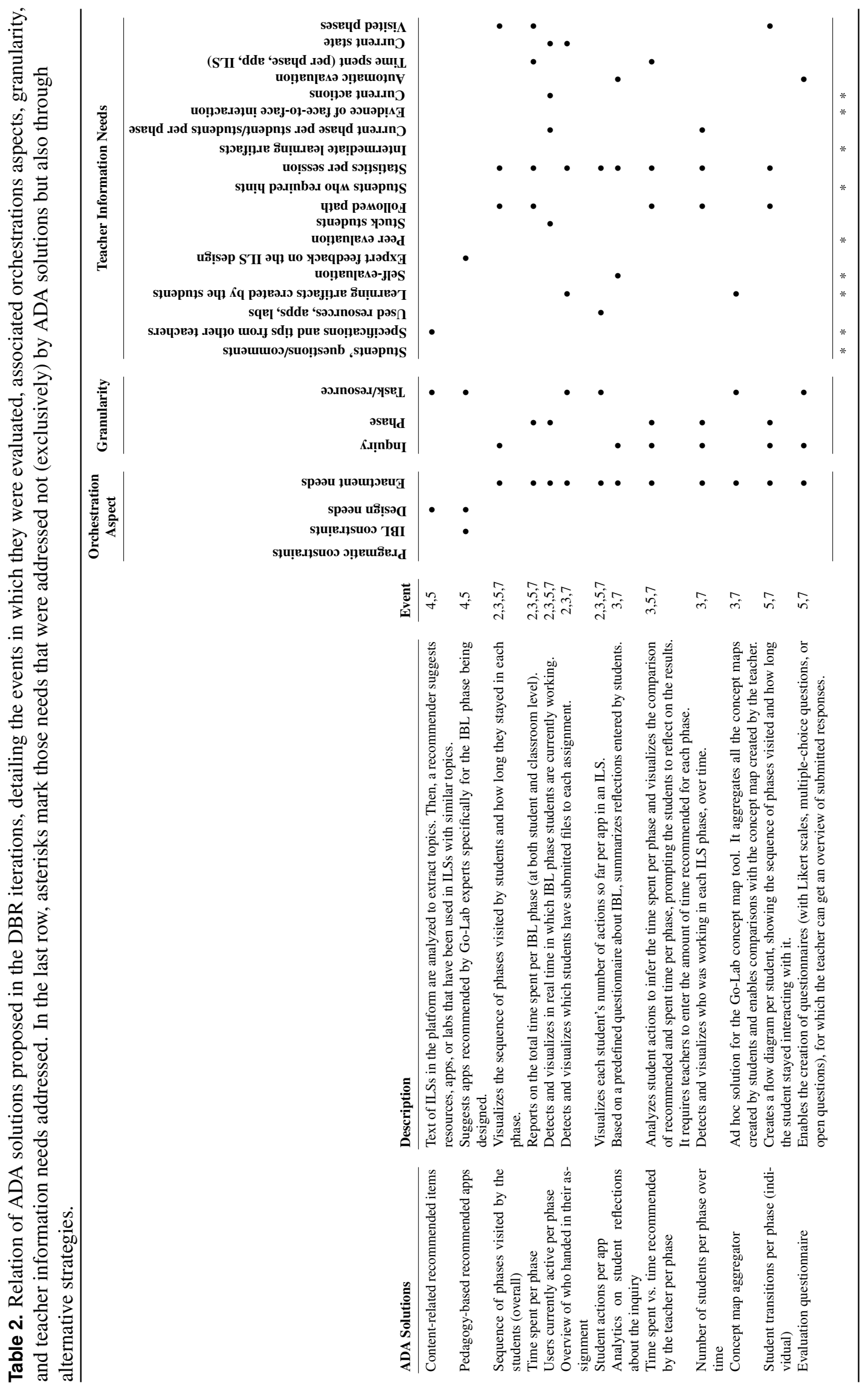



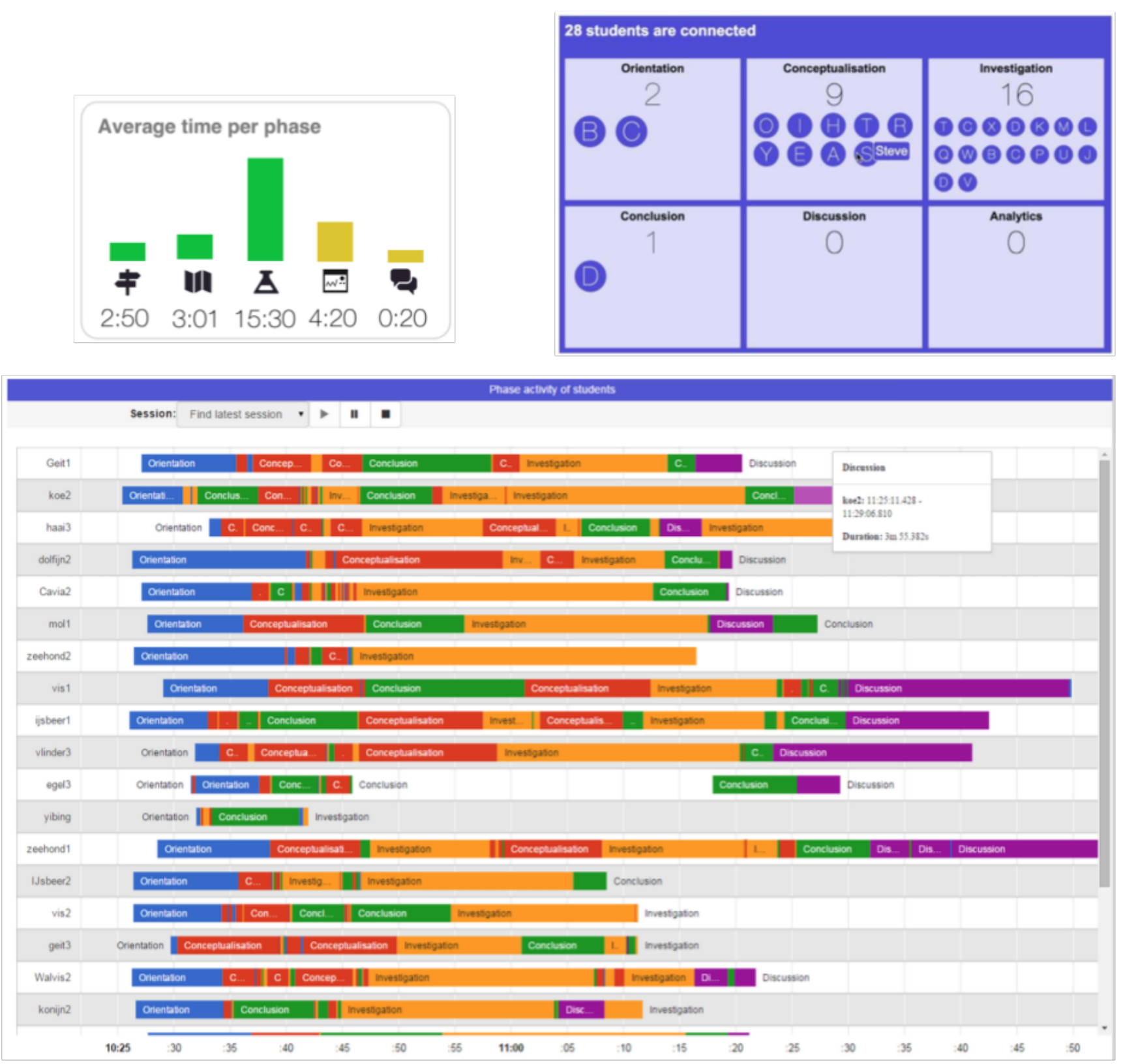

Figure 6. Example ADA solutions used by teachers during Iteration 2: "average time spent per phase," as used in the pre-enactment interview of Event_2 (top left); "users currently active per phase" (top right); and the "sequence of phases visited by the students," used during the actual Event_2 enactments (bottom).

assessment, and reflection (see Table 3). The enactment aspects were reduced to those three, since most of the needs reported in Event_1 and Event_3 (see Table 1) were related to them. Then, two teachers decided to use three of these solutions in the classroom. We observed all eight sessions to collect emerging teacher needs and understand how the implemented ADA solutions were integrated into teaching practice. Afterwards, teachers were interviewed about the problems they had faced and the usefulness of the solutions. Descriptive statistics were used on the quantitative feedback (about the perceived usefulness), also extracting quotes from the interviews about orchestration needs and other tool suggestions. Observations helped detect emergent orchestration needs, which were later cross-checked with teachers themselves during the interviews.

In line with results from the previous iteration, all three teachers expressed that they were mainly interested in the learning process and in student-generated artifacts, mostly for assessment purposes. All three teachers asked experts or peers for advice on apps/labs adequate for their pedagogical objectives and requested feedback to refine their ILSs. When informed about the 
Table 3. Perceived usefulness (from interviews with teachers) and actual use (from researcher observations) of ADA solutions in the authentic classroom inquiry enactments (Event_2). The numeric value of the perceived usefulness (score column) is the average rating by the teachers $(n=3)$ of usefulness for different purposes (fourth column), given in a seven-point Likert scale from Strongly Disagree (-3) to Strongly Agree (+3).

\begin{tabular}{|c|c|c|c|c|c|c|}
\hline \multicolumn{7}{|c|}{ Event 2 (Enactments, $n=3)$} \\
\hline \multicolumn{3}{|c|}{ ADA Solution } & \multicolumn{2}{|l|}{ 1st Interview $(n=3)$} & \multirow{2}{*}{$\begin{array}{c}\text { Observation } \\
\text { Teachers who } \\
\text { actually used } \\
\text { the app }\end{array}$} & \multirow{2}{*}{$\begin{array}{l}\text { 2nd Interview }(n=3) \\
\text { Sample of qualitative } \\
\text { feedback }\end{array}$} \\
\hline $\begin{array}{c}\text { Orchestration } \\
\text { Aspect }\end{array}$ & Granularity & $\begin{array}{l}\text { ADA } \\
\text { Analyses }\end{array}$ & $\begin{array}{l}\text { I would use this information for } \\
\ldots\end{array}$ & $\begin{array}{c}\text { Score }[-3,3] \\
(\bar{x}, s)\end{array}$ & & \\
\hline \multirow{5}{*}{ Enactment } & \multirow{5}{*}{ Inquiry } & \multirow{5}{*}{$\begin{array}{l}\text { Sequence of } \\
\text { phases visited } \\
\text { by the } \\
\text { students } \\
\text { (overall) }\end{array}$} & awareness in the classroom & $-1.33,2.08$ & \multirow{5}{*}{0} & \multirow{5}{*}{$\begin{array}{l}\text { After seeing students going } \\
\text { back and forth between } \\
\text { phases during the enactment, } \\
\text { teachers were interested in } \\
\text { analyzing the "learning path" } \\
\text { to improve the ILS flow } \\
\text { description. }\end{array}$} \\
\hline & & & awareness out of the classroom & $-1.33,2.08$ & & \\
\hline & & & assessment & $-2.33,0.58$ & & \\
\hline & & & reflection on the learning process & $2.00,1.00$ & & \\
\hline & & & reflection on the learning design & $2.67,0.58$ & & \\
\hline \multirow{5}{*}{ Enactment } & \multirow{5}{*}{ Phase } & \multirow{5}{*}{$\begin{array}{l}\text { Time spent } \\
\text { per phase }\end{array}$} & awareness in the classroom & $-2.33,1.15$ & \multirow{5}{*}{ Alice, Bob } & \multirow{5}{*}{$\begin{array}{l}\text { "It could be a relevant } \\
\text { reflection indicator for better } \\
\text { understanding the students" } \\
\text { progress." }\end{array}$} \\
\hline & & & awareness out of the classroom & $-1.33,2.89$ & & \\
\hline & & & assessment & $-0.67,2.52$ & & \\
\hline & & & reflection on the learning process & $2.67,0.58$ & & \\
\hline & & & reflection on the learning design & $2.67,0.58$ & & \\
\hline \multirow{5}{*}{ Enactment } & \multirow{5}{*}{ Phase } & \multirow{5}{*}{$\begin{array}{l}\text { Users } \\
\text { currently } \\
\text { active per } \\
\text { phase }\end{array}$} & awareness in the classroom & $2.33,1.15$ & \multirow{5}{*}{ Alice, Bob } & \multirow{5}{*}{$\begin{array}{l}\text { "It supports teacher } \\
\text { awareness in both } \\
\text { face-to-face and distance } \\
\text { activities, triggering } \\
\text { interventions when some } \\
\text { unexpected behaviour } \\
\text { appears." }\end{array}$} \\
\hline & & & awareness out of the classroom & $3.00,0.00$ & & \\
\hline & & & assessment & $0.00,2.65$ & & \\
\hline & & & reflection on the learning process & $2.33,1.15$ & & \\
\hline & & & reflection on the learning design & $2.67,0.58$ & & \\
\hline \multirow{5}{*}{ Enactment } & \multirow{5}{*}{$\begin{array}{l}\text { Task/ } \\
\text { resource }\end{array}$} & \multirow{5}{*}{$\begin{array}{l}\text { Overview of } \\
\text { who handed } \\
\text { in their } \\
\text { assignment }\end{array}$} & awareness in the classroom & $2.67,0.58$ & & \multirow{5}{*}{$\begin{array}{l}\text { While assignments were not } \\
\text { delivered through the ILSs, } \\
\text { according to the teachers, } \\
\text { having access to the artifacts } \\
\text { created by the students "could } \\
\text { be useful for awareness and } \\
\text { later assessment." }\end{array}$} \\
\hline & & & awareness out of the classroom & $2.33,0.58$ & 0 & \\
\hline & & & assessment & $0.67,3.21$ & & \\
\hline & & & reflection on the learning process & $-3.00,0.00$ & & \\
\hline & & & reflection on the learning design & $-3.00,0.00$ & & \\
\hline \multirow{5}{*}{ Enactment } & \multirow{5}{*}{$\begin{array}{l}\text { Task/ } \\
\text { resource }\end{array}$} & \multirow{5}{*}{$\begin{array}{l}\text { Student } \\
\text { actions per } \\
\text { app }\end{array}$} & awareness in the classroom & $-0.50,2.18$ & \multirow{5}{*}{ Alice, Bob } & \multirow{5}{*}{$\begin{array}{l}\text { "Understanding which apps } \\
\text { attract/discourage the students } \\
\text { could support the reflection on } \\
\text { the learning design and } \\
\text { refinement of future ones." }\end{array}$} \\
\hline & & & awareness out of the classroom & $-0.67,3.21$ & & \\
\hline & & & assessment & $-2.33,0.58$ & & \\
\hline & & & reflection on the learning process & $-3.00,0.08$ & & \\
\hline & & & reflection on the learning design & $-3.00,0.00$ & & \\
\hline
\end{tabular}

possibility of including the aforementioned ADA solutions, Alice and Bob decided to integrate several of them into their ILSs (users currently active per phase, time spent per phase, and student actions per app; see Table 3).

The teachers' classroom setting and orchestration style (as per our observations) were quite varied. In the two enactments by Alice, students worked individually or in groups, while she walked around answering questions. Since Alice was not close to her computer, she displayed the ADA solutions using the classroom projector so that she (and her students) could see the visualizations (Figure 7, top). In order to choose which group of students she should visit next, Alice looked at the "users currently active per phase" and visited those who, according to her expectations, were behind/ahead of schedule, or those not listed in any phase (meaning they were not interacting with the ILS). Students periodically checked the projection to compare their own progress with that of their peers. In Bob's lessons, students worked in small groups sharing one computer. He monitored students from his desk using mainly the "users currently active per phase" and, occasionally, the "time spent per phase" (Figure 7, middle). This prompted him to access students' screens (using the school's classroom management software) in cases of inactive or delayed students, approaching students only when they had specific questions. In Chris's sessions, the 
students worked individually. Given that the classroom distribution enabled him to glance over each student's screen and experiments (Figure 7, bottom), he did not use any of the proposed ADA solutions.

Teachers had designed the ILSs to be used in 90-minute face-to-face sessions. However, in several cases students could not finish the activities on time or were absent during the session. Since Alice and Bob had added the ADA solutions a priori, they were able to monitor the students' online work as they accomplished the tasks later at home. Chris did not realize until he assessed the students' work that some inquiries were not complete-but it was too late to intervene.

After the enactment, a second interview was performed to reflect on the challenges that Alice, Bob, and Chris had faced during the study and to review their perceptions about the ADA solutions (right side of Table 1). Although the three teachers were mainly concerned about obtaining the learning artifacts produced by students during the learning process (e.g., hypotheses, concept maps, datasets and observations collected while interacting with a lab, or conclusions), they did not require any support in this regard, since all three asked students for paper-based reports about their work. Additionally, Alice and Chris took pictures of the reports and uploaded them in the institutional learning environment as a backup. Among the ADA solutions, the "time spent per phase" was considered especially useful for reflection, and the "users currently active per phase" was considered to be useful for awareness in both face-to-face and blended situations. The "student actions per app," not considered very relevant at the beginning, turned out to be useful to detect which resources had attracted/discouraged students (for reflection and refinement of the learning designs). Moreover, the fact that teachers had seen students going back and forth between phases raised their interest in analyzing the sequence of phases visited by the students (to improve the flow and descriptions of the ILSs they had designed). Also, while the students did not have to "hand in their assignments" through the ILS, the teachers' concern about accessing the learning artifacts highlights that just providing teachers with evidence about the learning process was insufficient. Teachers also provided suggestions on the solutions' usability, which led to their refinement.

\subsection{Event_3 (Expert Teacher Workshop 1)}

To prioritize the IBL orchestration needs of teachers and to perform a wider evaluation of the ADA solutions implemented so far, a two-hour workshop was held with expert teachers: 28 Go-Lab teachers (different from the ones participating in previous events) from 14 European countries were selected by the project partners as expert, pioneering teachers in implementing IBL.

During the workshop, teachers (in groups of four) had to rank and classify by degree of importance (high/medium/low) the list of orchestration needs emerging from Event_1. They were also invited to come up with additional needs not covered in the list (e.g., the last item in Table 1). Teachers also assessed to what extent such needs were satisfied at that moment in the Go-Lab platform (no $=-1$, partially $=0$, yes $=1$ ). Figure 8 (top) shows the results of this classification and assessment, whose quantitative analysis (averages and standard deviations) has been integrated in Table 1 (right-hand side). Interestingly, needs that appeared with low frequency in Event_1 were ranked as highly important in Event_3.

In the second part of the workshop, an extended set of ADA solutions for enactment at different granularity levels was introduced to the teachers (see screenshots in Figure 8). As described in Table 2, this new set contained the five solutions used in Event_2 (once they were refined in terms of usability) and four new solutions addressing other needs elicited in Event_1 and Event_2. To better understand the usefulness of the solutions, teachers were provided with textual descriptions and screenshots, and they also tested them out during the session. Afterwards, each group of four teachers evaluated the solutions using a seven-point Likert scale from Strongly Disagree $(-3)$ to Strongly Agree $(+3)$, for which we calculated averages and standard deviations, also providing open-ended perceptions and ideas for improvement (see Table 4).

The ADA solutions perceived as most useful were comparing actual versus teacher-recommended time spent per phase, analyzing students' reflections about the learning process, and reporting on which students handed in their assignments. Solutions related to timing were also highly valued: online users per phase, or time spent (per student or whole class). In terms of granularity, no special trend was identified.

The qualitative feedback highlighted that the usefulness of enactment-oriented ADA solutions depends on the teaching style (e.g., walking around the class vs. staying next to the computer). The main drawback identified was the limited accuracy of the solutions when ILSs contained third-party apps or labs that did not have activity tracking, which led to incomplete evidence of student activity. Also, teachers provided recommendations to improve the usability of solutions, which led to further refinements.

\subsection{Event_4 (Novice Teacher Workshop)}

To assess potential adoption barriers of newcomers to Go-Lab's IBL support, we organized a workshop on IBL for pre-service teachers in a master's program on secondary teacher education. Six participants attended the event $(n=6)$, none of whom had participated in previous events. This workshop targeted IBL and design-oriented orchestration needs (assuming those needs would be more acute for someone without prior experience teaching with inquiry methods). Given that the Go-Lab platform already guides the creation of the whole inquiry and its phases, the most demanding design task is to decide which content should appear in each phase. Thus, the goal of the ADA solutions tested in this event was to support such decisions (at the 

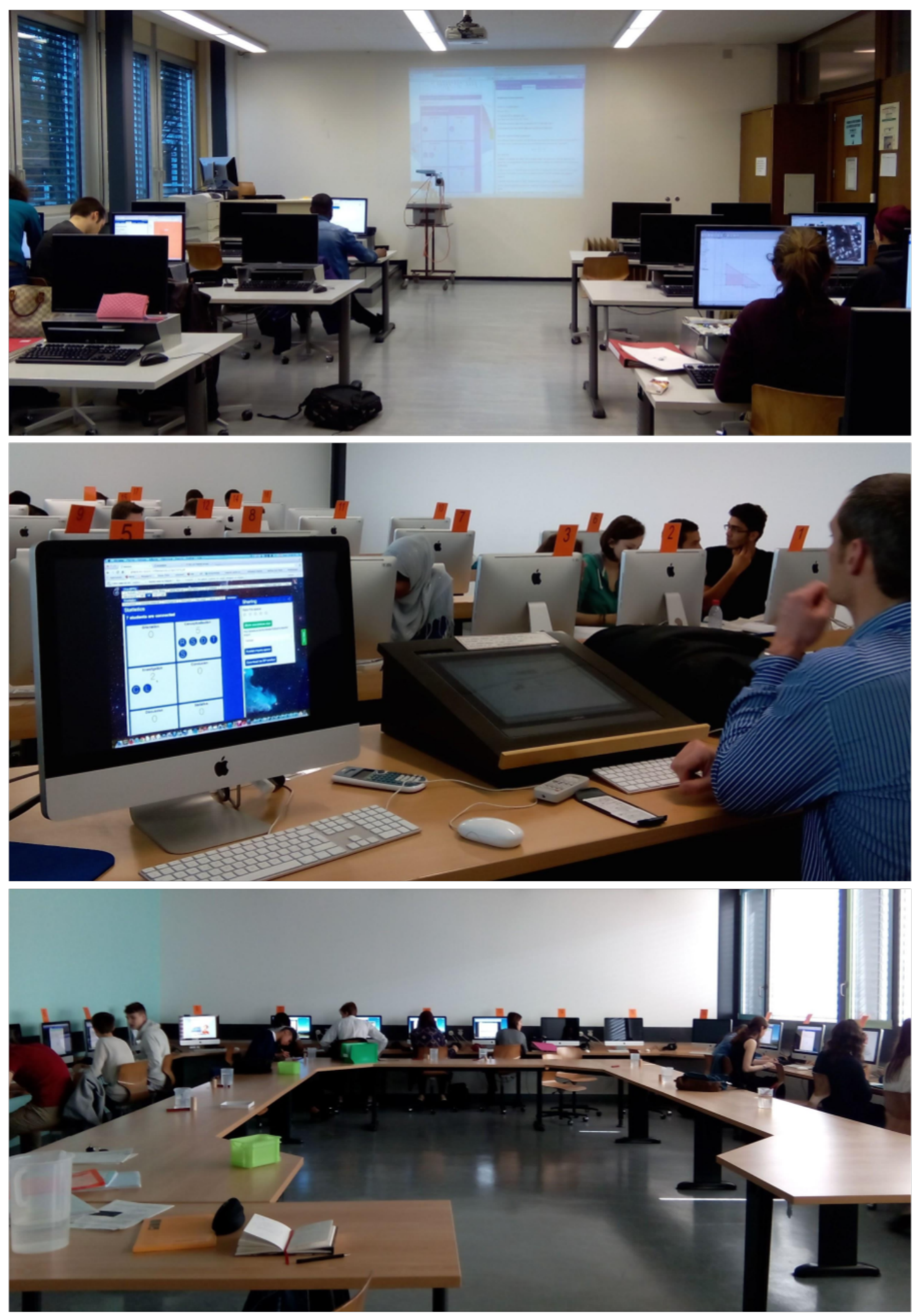

Figure 7. Classroom situations in the authentic enactment studies (Event_2). Awareness information displayed for Alice and students (top). Bob monitoring the students from his desktop (middle). The classroom physical distribution in Chris's cases allowed him to have an overview of students' activity by staying in the centre of the classroom (bottom). 
Table 4. Perceived usefulness of ADA solutions in the expert teacher workshop (Event_3)

\begin{tabular}{|c|c|c|c|c|}
\hline \multicolumn{3}{|c|}{ ADA Solution } & \multicolumn{2}{|r|}{ Event_3 ( $n=7$ groups, 28 teachers in total) } \\
\hline $\begin{array}{l}\text { Orchestration } \\
\text { Aspect }\end{array}$ & Granularity & ADA Analyses & $\begin{array}{c}\text { Perceived } \\
\text { Usefulness } \\
{[-3,3](\bar{x}, s)}\end{array}$ & Sample of Qualitative Feedback \\
\hline $\begin{array}{l}\text { Enactment- } \\
\text { assessment/ } \\
\text { reflection }\end{array}$ & Inquiry & $\begin{array}{l}\text { Sequence of phases } \\
\text { visited by students }\end{array}$ & $1.57,2.43$ & "The graphs need to be easier to read/understand." \\
\hline $\begin{array}{l}\text { Enactment- } \\
\text { awareness/ } \\
\text { assessment/ } \\
\text { reflection }\end{array}$ & Phase & Time spent per phase & $1.29,0.38$ & $\begin{array}{l}\text { "We cannot trust how much time students spend in a phase. } \\
\text { E.g.: if a student is working at home and takes a break and } \\
\text { time spent keeps on counting." }\end{array}$ \\
\hline $\begin{array}{l}\text { Enactment- } \\
\text { awareness }\end{array}$ & Phase & $\begin{array}{l}\text { Users currently active } \\
\text { per phase }\end{array}$ & $1.57,0.38$ & $\begin{array}{l}\text { "Usefulness depends on the type of teacher. If you are walking } \\
\text { around the class looking at the entire class, you cannot monitor } \\
\text { anything." }\end{array}$ \\
\hline $\begin{array}{l}\text { Enactment- } \\
\text { awareness/ } \\
\text { assessment }\end{array}$ & Task/resource & $\begin{array}{l}\text { Overview of who } \\
\text { handed in their } \\
\text { assignment }\end{array}$ & $2.86,1.11$ & "Enable filtering by user and assignment preview." \\
\hline $\begin{array}{l}\text { Enactment- } \\
\text { awareness/ } \\
\text { assessment/ } \\
\text { reflection }\end{array}$ & Task/resource & $\begin{array}{l}\text { Student actions per } \\
\text { app }\end{array}$ & $-0.14,2.16$ & "The app provides too much information at the same time." \\
\hline $\begin{array}{l}\text { Enactment- } \\
\text { assessment/ } \\
\text { reflection }\end{array}$ & Inquiry & $\begin{array}{l}\text { Analytics on student } \\
\text { reflections about the } \\
\text { inquiry }\end{array}$ & $2.29,0.53$ & $\begin{array}{l}\text { "Useful for teachers and students." "It helps teachers to collect } \\
\text { information about the students." "The questions should be } \\
\text { configurable." }\end{array}$ \\
\hline $\begin{array}{l}\text { Enactment- } \\
\text { assessment/ } \\
\text { reflection }\end{array}$ & Inquiry, phase & $\begin{array}{l}\text { Time spent vs. time } \\
\text { recommended by the } \\
\text { teacher per phase }\end{array}$ & $2.29,0.49$ & "It is not easy to suggest the amount of time." \\
\hline $\begin{array}{l}\text { Enactment- } \\
\text { assessment/ } \\
\text { reflection }\end{array}$ & Inquiry, phase & $\begin{array}{l}\text { Number of students } \\
\text { per phase over time }\end{array}$ & $-1.14,1.40$ & $\begin{array}{l}\text { "The app is not easy to understand. It required previous } \\
\text { explanation." }\end{array}$ \\
\hline $\begin{array}{l}\text { Enactment- } \\
\text { assessment/ } \\
\text { reflection }\end{array}$ & Task/resource & $\begin{array}{l}\text { Concept map } \\
\text { aggregator }\end{array}$ & $0.86,1.35$ & "It is a good idea [...] but it requires previous explanation." \\
\hline
\end{tabular}

task/resource granularity level) with pedagogical and content-based recommendations. In this case the proposed solutions use data about the learning designs (i.e., the ILSs), not about the learners. By analyzing the textual contents and apps used per phase in existing ILSs, this new set of ADA solutions suggested related apps, either according to the inquiry phase being edited or on the basis of the text and materials present in the ILS. Figure 9 shows the recommendations provided to the investigation phase of one example ILS.

The workshop spanned four hours and included an introduction to IBL and Go-Lab, a hands-on session on using an ILS as a student, and tasks for the creation of an ILS as a teacher. At the end of the session, using a survey with both closed-ended (Likert) and open-ended questions, participants were asked about their disposition toward the design-oriented ADA solutions.

Table 5 summarizes participants' answers, presenting the average and standard deviation of the quantitative data, and quotes from the open-ended questions. The results show a marginal difference in favour of pedagogy-based recommendations. In the qualitative feedback, participants pointed out that their low expertise in the platform conditioned their answers about the ADA solutions. Additionally, the low relevance of certain recommended items and the lack of awareness about the rationale behind the recommendations conditioned participants' trust in such recommendations.

\subsection{Iteration 2 Summary}

Overall, this iteration elicited several lessons (see Figure 5). In relation to IBL orchestration needs (RQa), as the initially important enactment needs were progressively supported by Go-Lab, design-related ones (e.g., getting feedback from experts and hints from experienced peers) gradually became more prominent. Also, the enactment case studies highlighted the crucial influence of classroom space and teaching style on the usage and adoption of ADA solutions. Unexpected blended learning may often occur, even in lessons planned to be face to face, which led us to think that there could be cases of blended learning among teachers' reporting of face-to-face usage of ILSs (the $47.62 \%$ of teachers using ILSs totally in the classroom, in Event_1). This 


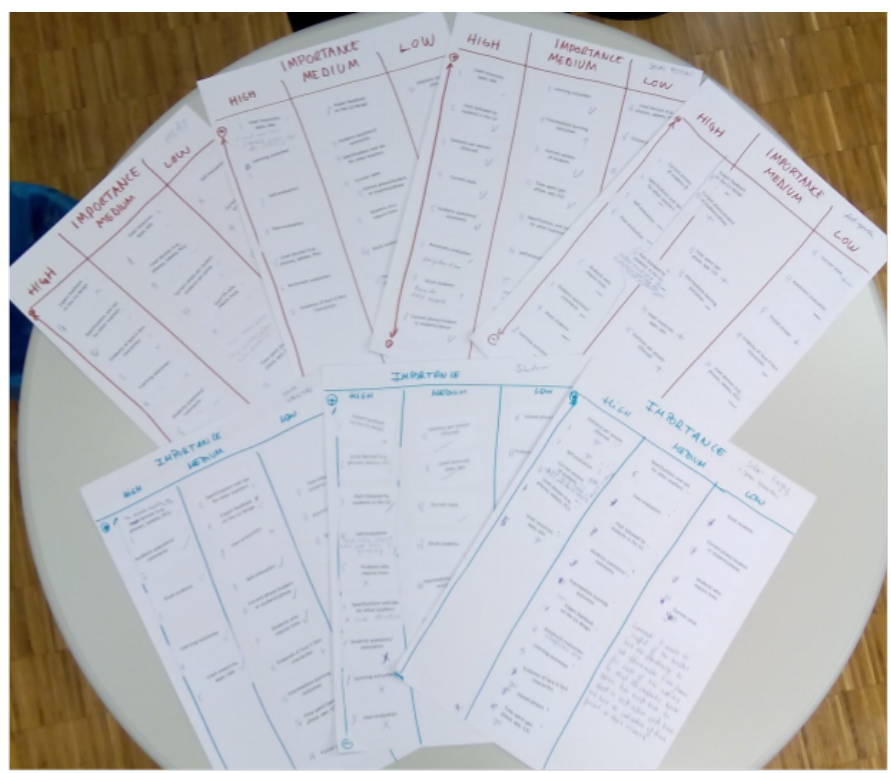

\section{Student actions per app}

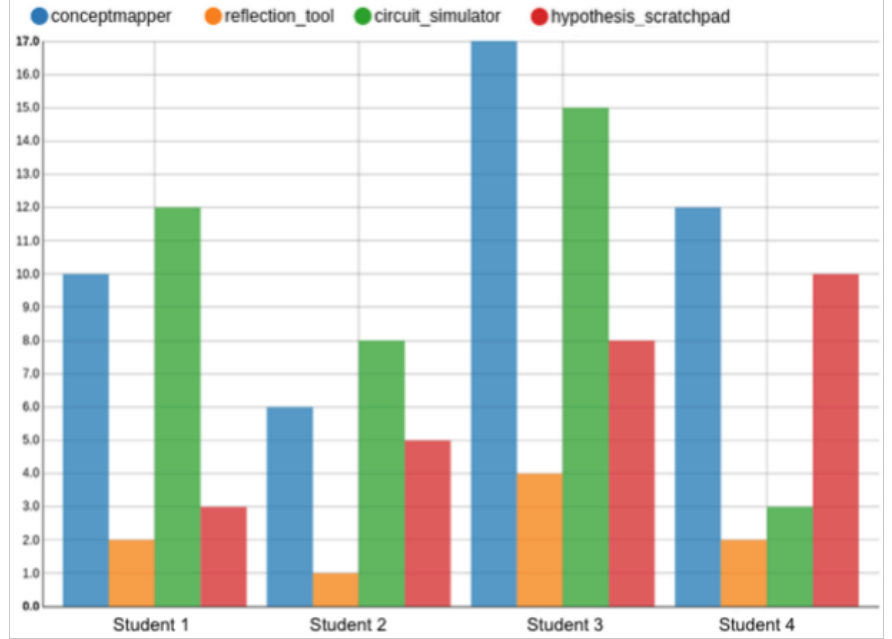

Number of students per phase over time

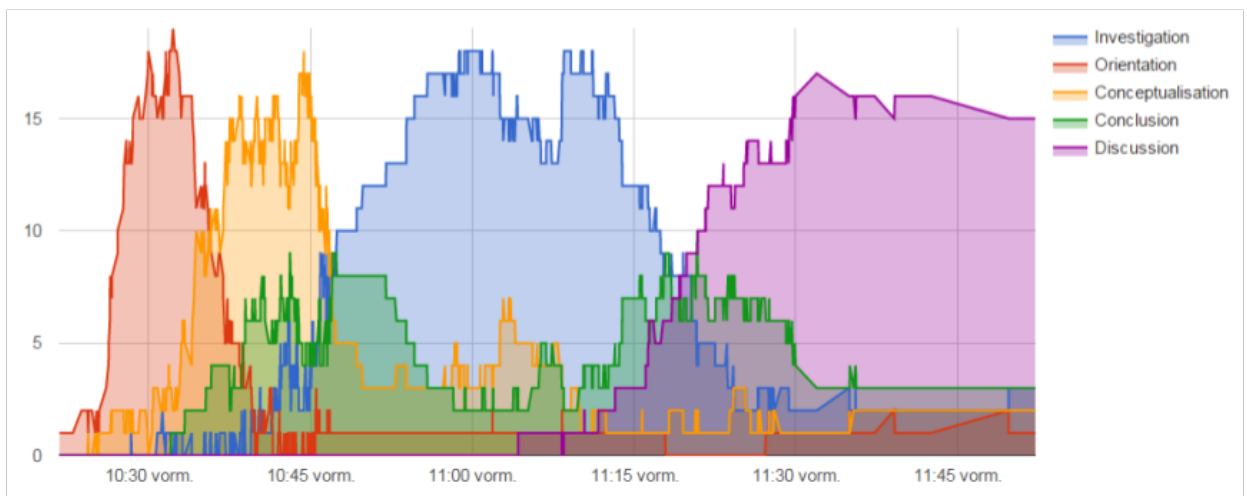

Figure 8. Images from the workshop with expert teachers (Event_3). Sheets generated by teachers with the prioritization of needs (top). Screenshots of ADA solutions evaluated in the second part of the event: "Student actions per app" (middle) and "Number of students per phase over time" (bottom). 


InVestigation $\checkmark$
Getting to know the constellations:
Step I - Using a planetarium software determine the dates the Sun enter
and exit the different constellations. Verify how many constellations are
crossed along the imaginary path of the Sun across the sky. Finally
compare the dates you found with the ones defined for the astrological
zodiac. Are they different? Why?
(If you are new on the use of the WWT you can find a nice tutorial here:
http://www.worldwidetelescope.org/
and here
http://wwtambassadors.org/page-type/tutorialvideo )
$\begin{aligned} & +\quad \text { Name } \nabla \\ & \text { Stellarium.graasp }\end{aligned}$
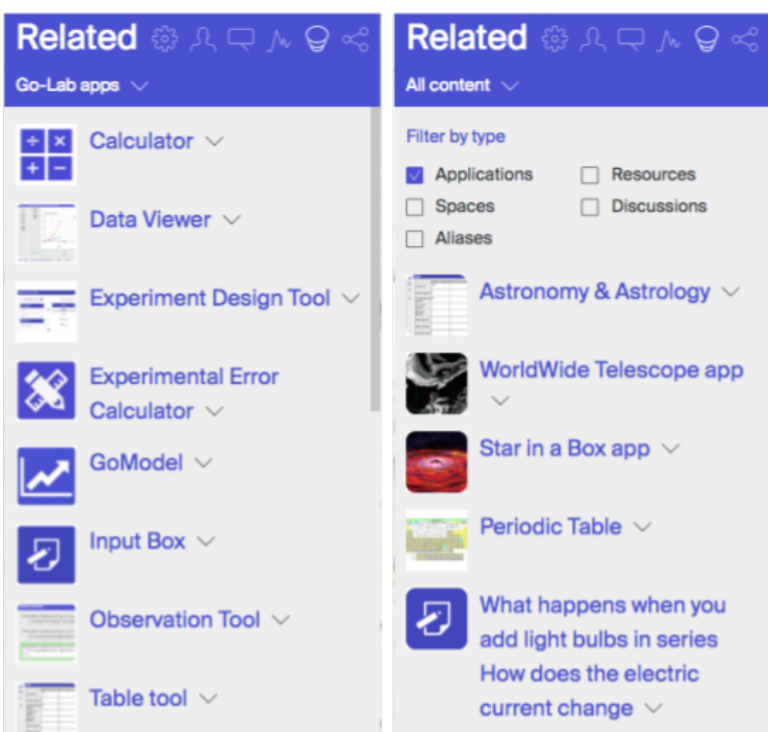

Figure 9. Screenshots of design-oriented ADA solutions. On the left, current contents of an investigation phase. In the middle, pedagogical recommendations based on the inquiry phase being edited (Go-Lab apps). On the right, app recommendations based on the astronomy-related content of the ILS.

Table 5. Evaluation of perceived usefulness of ADA solutions by pre-service teachers (Event_4). The five-point Likert scale goes from Strongly Disagree $(-2)$ to Strongly Agree $(+2)$.

\begin{tabular}{|c|c|c|c|c|c|}
\hline \multicolumn{3}{|c|}{ ADA Solution } & \multicolumn{3}{|r|}{ Event_4 (Workshop, $n=6)$} \\
\hline $\begin{array}{l}\text { Orchestration } \\
\text { Aspect }\end{array}$ & Granularity & ADA Analyses & Statement & $\begin{array}{l}\text { Score } \\
{[-2,2](\bar{x}, s)}\end{array}$ & Sample of Qualitative Feedback \\
\hline \multirow{2}{*}{ Design } & \multirow{2}{*}{ Task/resource } & \multirow{2}{*}{$\begin{array}{l}\text { Content-related } \\
\text { recommended } \\
\text { items }\end{array}$} & I found it useful & $0.50,0.84$ & \multirow{2}{*}{$\begin{array}{l}\text { "The algorithm provided recommendations that were not } \\
\text { relevant to the content." }\end{array}$} \\
\hline & & & $\begin{array}{l}\text { I'd like to use it } \\
\text { frequently }\end{array}$ & $0.50,0.55$ & \\
\hline \multirow{2}{*}{ IBL, design } & \multirow{2}{*}{ Task/resource } & \multirow{2}{*}{$\begin{array}{l}\text { Pedagogy-based } \\
\text { recommended } \\
\text { apps }\end{array}$} & I found it useful & $1.00,0.63$ & \multirow{2}{*}{$\begin{array}{l}\text { "To profit from the recommendations it would be } \\
\text { necessary to be more familiar with the platform. Before } \\
\text { starting to think about the content, I need to learn the } \\
\text { basic functionalities to reduce the cognitive load." }\end{array}$} \\
\hline & & & $\begin{array}{l}\text { I'd like to use it } \\
\text { frequently }\end{array}$ & $0.50,1.38$ & \\
\hline
\end{tabular}

increases the interest of the awareness support provided by ADA solutions. The results from Event_3 suggest that the proposed ADA solutions could contribute to addressing elicited teacher needs (see Table 1).

Regarding the suitability of the ADA solutions (RQb), we experienced the challenge of including (and designing the technological platform for) third-party apps and labs created beyond the project: while the increased variety of tools is beneficial when designing inquiries, it is a challenge for analytics due to the lack (or the heterogeneity) of student activity data from these tools. The workshop with pre-service teachers led to inconclusive results regarding how to support practitioners at design time using recommendations. It would be necessary to refine the recommendation algorithms (e.g., increase the accuracy of the results), to extend them (e.g., enable recommendations beyond the content available in the platform, or take social feedback into account), and/or to explore other inquiry design support. Finally, Event_2 and Event_3 confirmed the importance teachers ascribe to accessing learner-generated artifacts (initially raised during Event_1). Thus, several functionalities were developed (during and after this iteration) to enable teachers to access and export such artifacts.

\section{DBR Iteration 3}

The results from the previous iteration underlined that certain orchestration needs detected at the outset were now fully or partially addressed (see Table 1) and prompted the following question related to RQa: are there additional, emergent orchestration needs impeding the adoption of IBL? Furthermore, regarding RQb, we aimed at evaluating the fulfillment of orchestration needs and tried to investigate the actual adoption of Go-Lab's ADA solutions (as opposed to the perceptions and opinions of a limited number of teachers). The decisions and outcomes of this iteration are summarized in Figure 5. 


\subsection{Event_5 (Expert Teacher Workshop 2)}

At this point, the technological ecosystem (i.e., the repository and authoring platform) had evolved noticeably since the previous iterations, and the ADA solutions had been extended and refined according to the suggestions and usability feedback collected during Iteration 2. A two-hour workshop was held with 34 teachers from different European countries who had implemented ILSs in their own classrooms ( 22 of whom had never participated in previous events). To assess the added value of the ADA solutions, participants first received training and guidance about using such solutions within an ILS. Later, teachers integrated the ADA solutions in ILSs already created and used by the teachers in their own classrooms. This exercise enabled the participants to analyze, interpret, and visualize the work done by the students in the context of their own lessons. At the end of the workshop, teachers answered Likert-scale questions about the usefulness and expected frequency of use of the ADA solutions. Additionally, through a questionnaire and a focus group, teachers were asked about how the Go-Lab ecosystem and the ADA solutions addressed their design and enactment needs (in terms of awareness, assessment, and reflection - the main orchestration aspects related to the needs reported in Event_1 and Event_3), and which ones were not yet covered. Quotes from the focus group and teachers' open-ended written feedback were clustered by topic.

All ADA solutions were perceived as useful (averages ranging from 1.24 to 1.52 , in a [-2,2] scale; see Table 6). Interestingly, responses about the expected frequency of use were lower (averages ranging from 0.90 to 1.34) and more varied. While there was no exact matching in the ranking of usefulness and expected adoption, the differences in the scores were small. Interestingly, while in previous events teachers had requested a customizable version of the solution "Analytics on student reflections about the inquiry," the solution proposed to address this request (the "Evaluation questionnaire") received the lowest scores in terms of usefulness and expected use. As per the qualitative feedback, such results could be linked to bugs found by the teachers while testing the tool.

Table 6. Perceived usefulness and expected frequency of use of ADA solutions by Go-Lab teachers (Event_5)

\begin{tabular}{|c|c|c|c|c|c|c|}
\hline \multicolumn{3}{|c|}{ ADA Solution } & \multicolumn{4}{|c|}{ Event_5 (Workshop, $n=34)$} \\
\hline $\begin{array}{l}\text { Orchestration } \\
\text { Aspect }\end{array}$ & Granularity & ADA Analyses & Statement & $\begin{array}{c}\text { Score } \\
{[-2,2](\bar{x}, s)}\end{array}$ & $\begin{array}{c}\text { Orchestration } \\
\text { Need Satisfaction } \\
{[-2,2](\bar{x}, s)}\end{array}$ & $\begin{array}{l}\text { Sample of Qualitative Feedback } \\
\text { about Orchestration Needs }\end{array}$ \\
\hline \multirow{4}{*}{ Design } & \multirow{2}{*}{ Task/resource } & \multirow{2}{*}{$\begin{array}{l}\text { Pedagogy-based } \\
\text { recommended apps }\end{array}$} & I think it is useful & $1.35,0.81$ & \multirow{4}{*}{$1.29,0.76$} & "Indeed, we need help to find more \\
\hline & & & I will use it often & $1.12,0.84$ & & $\begin{array}{l}\text { suitable online labs for math, biology, } \\
\text { chemistry ICT or computer science." }\end{array}$ \\
\hline & \multirow{2}{*}{ Task/resource } & \multirow{2}{*}{$\begin{array}{l}\text { Content-related } \\
\text { recommended items }\end{array}$} & I think it is useful & $1.44,0.86$ & & "It would be useful to have expert \\
\hline & & & I will use it often & $1.09,0.90$ & & recommendations or ILS rankings.” \\
\hline \multirow{6}{*}{$\begin{array}{l}\text { Enactment- } \\
\text { awareness }\end{array}$} & \multirow{2}{*}{ Task/resource } & \multirow{2}{*}{$\begin{array}{l}\text { Student actions per } \\
\text { app }\end{array}$} & I think it is useful & $1.24,0.91$ & \multirow{6}{*}{$1.07,1.07$} & \multirow{6}{*}{$\begin{array}{l}\text { "We need solutions for offline } \\
\text { learning. So far it's internet } \\
\text { dependent: no internet, no lesson." }\end{array}$} \\
\hline & & & I will use it often & $1.03,0.87$ & & \\
\hline & \multirow{2}{*}{ Inquiry, phase } & \multirow{2}{*}{ Time spent per phase } & I think it is useful & $1.38,0.78$ & & \\
\hline & & & I will use it often & $1.00,0.96$ & & \\
\hline & \multirow{2}{*}{ Inquiry, phase } & \multirow{2}{*}{$\begin{array}{l}\text { Users currently } \\
\text { active per phase }\end{array}$} & I think it is useful & $1.41,0.78$ & & \\
\hline & & & I will use it often & $1.14,0.95$ & & \\
\hline \multirow{12}{*}{$\begin{array}{l}\text { Enactment- } \\
\text { assessment/ } \\
\text { reflection }\end{array}$} & \multirow{2}{*}{ Inquiry, phase } & \multirow{2}{*}{$\begin{array}{l}\text { Student transitions } \\
\text { per phase } \\
\text { (individual) }\end{array}$} & I think it is useful & $1.31,0.81$ & \multirow{12}{*}{$1.34,0.72$} & \multirow{12}{*}{$\begin{array}{l}\text { "Give an automatic statistical } \\
\text { summary of what students have been } \\
\text { doing." "It takes a lot of work to } \\
\text { evaluate all the students' } \\
\text { work." "Enable ILSs to be printed, so } \\
\text { that I can evaluate the student work } \\
\text { offline." "Embedded apps for pre-test } \\
\text { and post-test." "Survey [evaluation } \\
\text { questionnaire] not working." }\end{array}$} \\
\hline & & & I will use it often & $1.10,0.90$ & & \\
\hline & \multirow{2}{*}{ Inquiry, phase } & \multirow{2}{*}{$\begin{array}{l}\text { Sequence of phases } \\
\text { visited by students } \\
\text { (overall) }\end{array}$} & I think it is useful & $1.34,0.81$ & & \\
\hline & & & I will use it often & $1.17,0.85$ & & \\
\hline & \multirow{2}{*}{ Inquiry, phase } & \multirow{2}{*}{$\begin{array}{l}\text { Time spent vs. time } \\
\text { recommended by the } \\
\text { teacher per phase }\end{array}$} & I think it is useful & $1.34,0.77$ & & \\
\hline & & & I will use it often & $1.03,0.91$ & & \\
\hline & \multirow{2}{*}{ Inquiry, phase } & \multirow{2}{*}{ Time spent per phase } & I think it is useful & $1.52,0.69$ & & \\
\hline & & & I will use it often & $1.34,0.72$ & & \\
\hline & \multirow{2}{*}{ Task/resource } & \multirow{2}{*}{$\begin{array}{l}\text { Student actions per } \\
\text { app }\end{array}$} & I think it is useful & $1.34,0.90$ & & \\
\hline & & & I will use it often & $1.07,0.84$ & & \\
\hline & \multirow{2}{*}{$\begin{array}{l}\text { Inquiry, } \\
\text { task/resource }\end{array}$} & \multirow{2}{*}{$\begin{array}{l}\text { Evaluation } \\
\text { questionnaire }\end{array}$} & I think it is useful & $1.28,0.88$ & & \\
\hline & & & I will use it often & $0.90,1.01$ & & \\
\hline
\end{tabular}

Participant teachers were especially satisfied with the enactment-oriented assessment and reflection support $(\bar{x}=1.34)$, 
including not only the ADA solutions but also other functionalities, like the support for student reflection and self-evaluation. In this regard, during the focus group teachers reported that the main unfulfilled needs referred to the general problem of assessment (e.g., supporting the automatic assessment of student learning artifacts), some of which could easily be supported by learning analytics solutions (e.g., using automatically processed pre-/post-tests). Teachers were slightly less satisfied with the design-oriented support $(\bar{x}=1.29)$, including not only the ADA solutions (i.e., the recommenders from Iteration 2 above) but also the community support, online materials, and pedagogical guidance. In the focus group, teachers emphasized the importance of supporting the discovery of apps, labs, and other resources and mentioned the potential usefulness of having not only content-related but also expert recommendations and socially ranked ILSs. Other common limitations of TEL solutions, such as the need for better internationalization, were pointed out as well. Enactment-related needs were considered less satisfactorily addressed $(\bar{x}=1.07)$, mainly due to bugs, usability issues, information and communications technology (ICT) infrastructure (lack of, or poor, WiFi), and student language barriers.

In summary, while teachers seemed satisfied with the ADA solutions, this event uncovered the fact that unmet orchestration needs were related to the particular ILS content, contextual constraints, or the general use of technology, rather than being IBL specific. Still, future ADA solutions could address some of these general needs. For example, at the task level, concept clouds automatically extracted from student productions could raise teacher awareness about whether students are internalizing the concepts of the ILS. Also, concept clouds could be applied at the inquiry or phase level, comparing the students' and the teacher's clouds (e.g., extracted from the ILS instructions). At the inquiry level, the relation between phases could also be analyzed, e.g., between the hypothesis, the observations, and the conclusions entered by the students.

\subsection{Event_6 (Expert Teacher Workshop 3)}

In order to uncover the remaining or emergent IBL orchestration challenges hindering the adoption of Go-Lab, we organized a workshop with 22 teachers who had previous expertise, both with IBL and Go-Lab ( 9 of them had already participated in previous events). During the workshop, part of a teacher-training program on innovative practice using IBL, teachers reflected on and discussed the difficulties they still faced when integrating IBL and Go-Lab in their practice. This was done as a focus group, where teachers expressed benefits and outstanding orchestration challenges (see Figure 10, top). The recording of the session and the artifacts produced by the teachers was analyzed in relation to the orchestration aspects depicted in Figure 3, clustering voiced difficulties per topic.

As we can see in Figure 10 (bottom), teachers cited classic challenges in TEL adoption, like insufficient ICT infrastructure and usability and language barriers. For this kind of pragmatic challenge, ADA solutions may not be of great help, since there is no specific relation either with the learning design or with learning and teaching practices.

Looking at IBL-specific challenges, we see that time restrictions (and the increased time that designing and enacting inquiries takes) were most often mentioned. Similarly, many of the other challenges relate to making the most of teachers' limited time: more findable, reusable ILSs and apps; assessment functionalities; etc. Additionally, certain remarks were made about the difficulties for teachers to provide adequate direction in the inquiries and for students to assume more active roles. ADA solutions could be especially valuable in addressing these IBL-specific challenges.

Several issues were specific to the Go-Lab ecosystem: teachers mentioned the (in)compatibility between Go-Lab and their already-existing technological classroom ecosystems (in terms of web browsers, hardware devices, etc.), or particular usability problems of Go-Lab. These barriers are beyond the scope of ADA solutions per se, but built-in compatibility tests could be explored to mitigate them.

\subsection{Event_7 (Platform Usage Analysis)}

In our DBR thus far we had collected opinions and perceptions and observed actual enactment practice from 95 teachers. Yet, this evidence could represent a skewed vision of the awareness and adoption of the ADA solutions for IBL orchestration offered by Go-Lab. The participants involved in the previous events represent less than $0.35 \%$ of the Go-Lab user base, and their commitment and motivation may not be representative of the rest of that population. To tap into the whole Go-Lab teacher community, we analyzed the overall usage of Go-Lab's ADA functionalities until 1 November 2018.

In order to understand the level of awareness and adoption of the ADA solutions, we analyzed the ILSs of every teacher registered in the platform (26,810 teachers, excluding partners from Go-Lab and related projects). For each teacher, we calculated (a) how many ILSs the teacher had (co)created in total, (b) how many ILSs included at least one ADA solution that each teacher had (co)created; (c) how many of the teacher's ILSs had potentially been implemented in the classroom ${ }^{7}$; and (d) how many ILSs with at least one ADA solution had potentially been implemented. Figure 11 shows the number of teachers

\footnotetext{
${ }^{7}$ To determine whether an ILS has been implemented with students on the basis of the platform's logs, the following heuristic was used, based on the fact that most teachers reported having 10 or more students per classroom (Event_1) and that an average European classroom may have around 20 students. Since teachers reported having used Go-Lab in small groups (i.e., two students may have used a single computer to access the ILS), we considered ILSs as potentially implemented when there were traces from at least 10 different students in an ILS.
} 

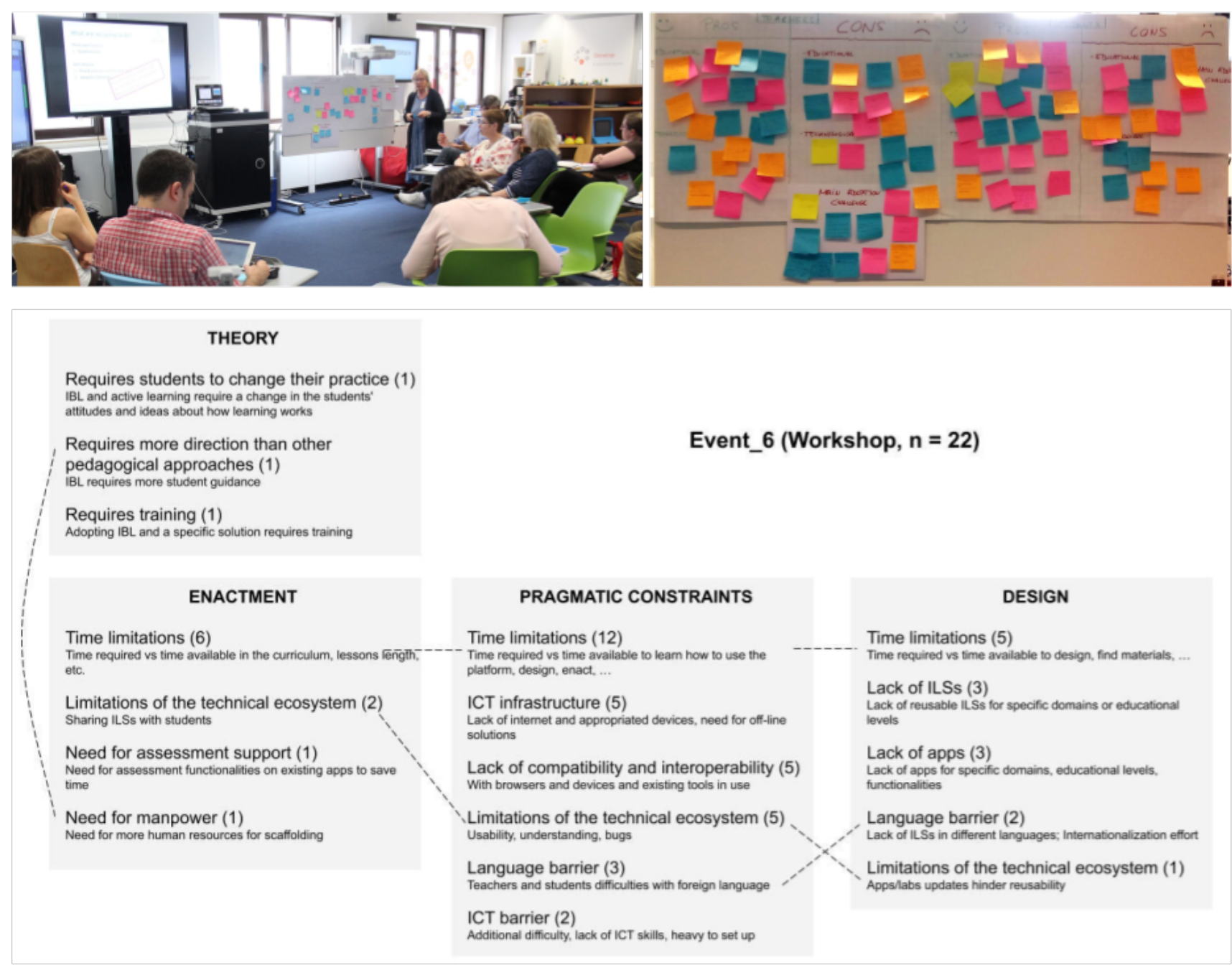

Figure 10. Images from a workshop with Go-Lab teachers (Event_6) (top), and main remaining IBL orchestration challenges from the analysis of workshop evidence (bottom). The number of teachers mentioning each challenge appears in parentheses. Lines connect related challenges.

who had ILSs corresponding to each of the four aforementioned categories. While the usage of ADA solutions to support the design could not be tracked (since they are built-in solutions that anyone can use at any time), the analysis focused on the rest of the apps listed in Table 2.

Regarding the awareness of ADA solutions, out of 15,894 teachers who (co)created at least one ILS, $25.07 \%$ included at least one of the ADA solutions. Out of 3,985 teachers that potentially implemented ILSs in their classroom, 69.62\% did so with at least one ILS featuring these solutions. Indeed, more than 50\% of those including ADA solutions in an ILS used them again in at least one other ILS of theirs (regardless of whether we look at co-created ILSs or ILSs potentially implemented in the classroom).

We can look at the numbers of teachers who have (co)created ILSs versus those who have gone on to implement them in their classrooms (Figure 11, left margin) as a sort of "conversion funnel" (Kotler \& Armstrong, 2010). Since it requires additional effort to put an ILS into practice in the classroom, only a portion of those who create ILSs will go on to become "implementers." Indeed, only $15.18 \%$ of the (co)creators become implementers of ILSs and, out of this group, $49.15 \%$ did it more than once. However, if we look at the teachers who included ADA solutions in their ILSs (right-hand margin in Figure 11), we find a much larger proportion $(42.16 \%$ ) that went on to become classroom implementers, and $57.62 \%$ of them did it with more than one ILS. Multiple factors can be at play here: is this because the ADA solutions indeed provide more adequate IBL orchestration support, or because of some other inherent trait of the teachers (which causes them to both try out ADA solutions and implement them in the classroom)? This observational study cannot answer this question, which can be the focus of future 
research.

These results align with the emphasis placed on enactment needs by the teachers participating in the previous DBR events (especially in the survey and workshops). Given the high proportion of teachers who repeatedly implement their ILSs using ADA solutions once they try them, one potential adoption promotion strategy could be to offer built-in guidance about the benefits that ADA solutions could have in a teacher's ILS, clearly explaining how these solutions can be integrated into their teaching practice.

(a) How many teachers have (co)created XILSs?

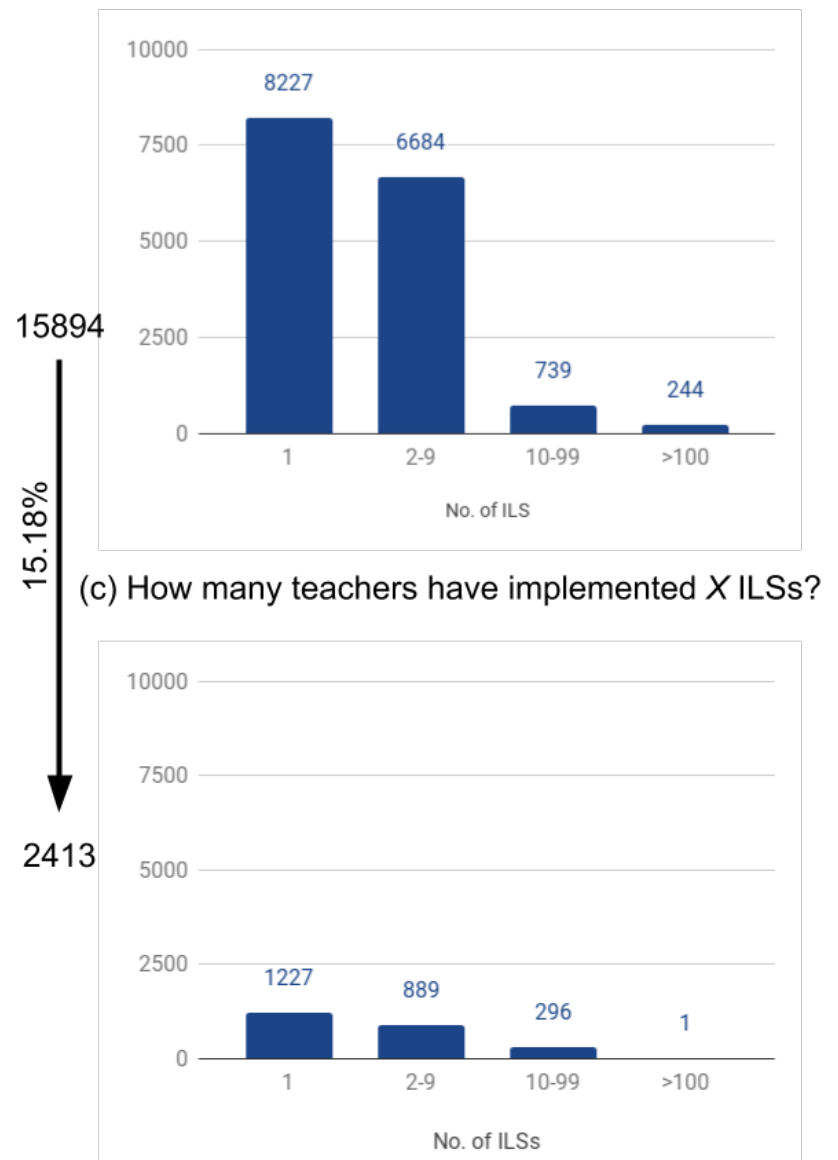

(b) How many teachers have (co)created $X$ ILSs with ADA solutions

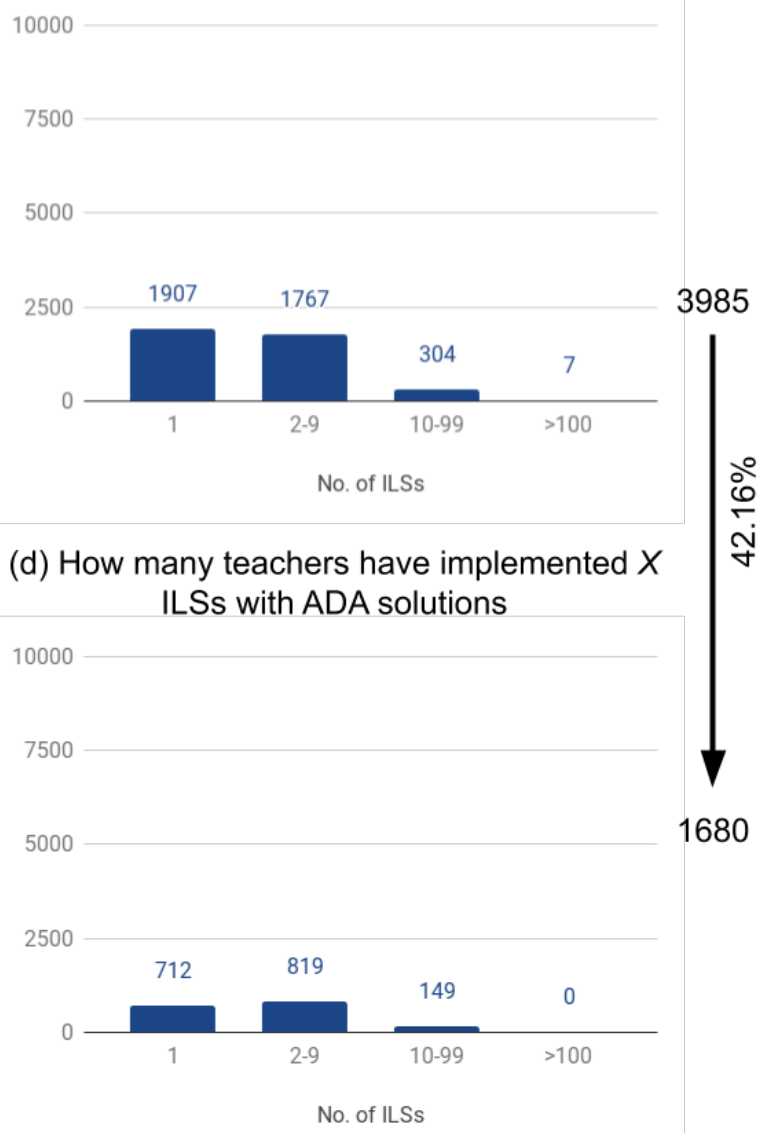

Figure 11. Platform usage statistics of awareness and adoption of the ADA solutions in Go-Lab, in terms of (co)created and implemented ILSs (Event_7). In the margins, the total number of teachers in each of the categories.

In terms of orchestration needs, this overall analysis suggests that many teachers (40.71\%) disengage before (co)creating an ILS in the platform. These results match with the feedback obtained from the pre-service teachers in Event_4. While some teachers may have just tried the Go-Lab ecosystem out of curiosity (without intending to adopt it later on), it could be useful to better integrate the ADA solutions into the design process (e.g., with a wizard guiding newcomers in the design process, based on pedagogically grounded recommendations and patterns observed from other teachers).

\subsection{Iteration 3 Summary}

Overall, this iteration provided us with an updated, more complete view of teachers' orchestration needs (RQa) when implementing IBL in their classrooms. Furthermore, in relation to RQb, the evaluation of the ADA solutions implemented in Go-Lab and their actual adoption helped us understand how to align learning design and analytics in a way that supports teacher orchestration (which could be distilled into design guidelines). These two outcomes are described in the following section.

\section{Discussion}

As shown in Figure 5, the three DBR iterations raised a number of findings regarding our research questions: (RQa) "What are the IBL orchestration needs of teachers?" and (RQb) "To what extent do ADA solutions fulfill those needs?" Below we 
synthesize these needs and design guidelines for ADA solutions devoted to IBL orchestration as main theoretical outputs from our DBR (Barab \& Squire, 2004).

\subsection{Teacher Needs in Orchestrating IBL and Suitability of ADA Solutions}

From the elicitation and prioritization of teacher needs performed throughout the data-gathering events, and our own observations during this whole DBR process, we can categorize the orchestration needs that teachers implementing IBL in their classrooms seem to have (RQa). We have classified these needs in Figure 12, according to the different orchestration aspects addressed in this research.

\section{When orchestrating IBL there is a need for ...}

\section{THEORY}

IBL training

Adopting IBL requires training

more direction

$\mathrm{IBL}$ requires more student scaffolding

fostering students to change their practice $\mathrm{IBL}$ and active learning require a change in the teacher and student practice

\section{DESIGN}

reduced design time

To design the lesson, find materials, ...

reusable designs (ILSs)

For specific domains or educational levels that can be later adapted according to the contextual, cultural and curricular constraints

\section{findable resources}

For specific domains, educational levels, functionalities

guidance and feedback

Need for IBL guidance and expert feedback

\section{PRAGMATIC CONSTRAINTS}

\author{
blended orchestration support \\ Time constraints or unexpected events may require \\ orchestrating blended learning
}

interpretable, actionable analysis

That provides meaningful and contextualised evidence of student learning

\section{compatibility and interoperability}

With browsers, devices and existing tools in use

\section{ENACTMENT}

\section{manpower \\ Need for more human resources for monitoring and regulating each learning path}

\section{awareness support}

Need for versatile visualizations of the student activity and current status usable with a variety of teaching management styles and classroom layouts

\section{assessment support}

Need for peer, self and automatic assessment solutions

reflection support

Need for deeper analyses about the learning process

Figure 12. Main IBL orchestration needs extracted during our DBR process.

From the perspective of $I B L$ theory, the teachers' perceived need for receiving feedback from more experienced peers and experts supports the importance of social practices for IBL adoption reported in other papers (de Jong et al., 2021; Rodríguez-Triana et al., 2020). Aligned with the existing literature (Blake et al., 2013; Sergis et al., 2019; Sharples, 2013), to help teachers guide students toward more active learning models, IBL pedagogical principles and best practices should be provided. While teacher requests tagged as IBL related were mentioned infrequently, they were closely linked to the most popular demand among teachers: to effectively guide students within the time constraints.

Among the needs for coping with (planned and unplanned) pragmatic constraints, the main concerns of the teachers referred to the interoperability and compatibility of the IBL platform with the technological ecosystem already in use and the need for contextualized and easily interpretable learning analytics solutions to help them monitor and understand the learning process. While the former concern about interoperability is common in TEL research (Ochoa \& Ternier, 2017), the latter is a 
well-known issue in the learning analytics community (Hernández-Leo et al., 2018). In addition, due to the student-driven nature of the inquiries, part of the learning activity may happen outside the classroom (and outside a controlled technological setting (Mulholland et al., 2012)). Thus, there is a clear need to make learning designs and technologies compatible with blended learning (Rodríguez-Triana et al., 2017).

As stated by other researchers (Emin-Martinez et al., 2014; Hernández-Leo et al., 2018; Lockyer \& Dawson, 2011; Lockyer et al., 2013) and illustrated in the different events of our DBR process, the alignment between learning design and analytics can aid design and enactment needs:

- Before the learning activity, ADA solutions can support the learning design, analyzing existing designs to make recommendations at the inquiry or app/resource level, thus reducing the complexity and workload of the task (see Event_4).

- During the learning activity (as seen in Event_2), ADA solutions could facilitate teachers' awareness needs, such as the constant search for struggling students, or raise awareness about situations that may require teacher intervention (e.g., excessive time deviations from the planned design). In this way, ADA can reduce the management and awareness load, freeing teachers' time and attention so that they better regulate, intervene in, and adapt the learning activities.

- After the learning activity, ADA solutions may provide analytics for assessment (e.g., automatic statistical summaries of the learning activities or pre-/post-tests) and help teachers reflect on their inquiries, and what parts of them need to be improved for future designs (as happened in Event_2).

Our second research question, about whether or not the ADA solutions implemented in Go-Lab fulfill teachers' IBL orchestration needs $(\mathrm{RQb})$, can be illuminated by drawing upon the evidence of Events 2 to 7 (following the four topics of analysis in Figure 3):

Design support The proposed recommender systems to aid in ILS design had mixed success. Their main target audience (novice teachers) valued both pedagogical and content-based recommendations positively but pointed out shortcomings in accuracy or considered them overwhelming (Event_4). More expert teachers, on the other hand, valued them quite highly (Event_5). Despite this mildly positive reaction, teachers also pointed out several unsolved challenges in order to make the design of ILSs faster and more versatile (Event_6).

Enactment support This area was the most complex in terms of number and variety of needs identified. Awareness solutions showing real-time data were well valued, especially those comparing the time spent with the lesson plan, and pointing to the students' current phase within the inquiry (Event_3). More complex visualizations (like the temporal evolution of students across inquiry phases), however, did not seem worth the increased load on teachers' attentional resources (Event_3). Enactment time limitations remain an unsolved problem for many teachers (Event_6). ADA solutions related to assessment and monitoring of assignments are considered important by teachers (Event_2, Event_3) but may not fully cover teachers' assessment needs yet (Event_6).

Support within pragmatic constraints The case studies of classroom enactment (Event_2) showed that ADA solutions were usable in a variety of authentic settings by different kinds of teachers. The fact that teachers' expected frequency of use is moderately high across all ADA solutions (Event_5) also hints at the tools complying with at least basic classroom contextual constraints. In general, ADA solutions have been adopted by thousands of teachers using the Go-Lab orchestration platform, even if the proportion of ADA users is still moderate (Event_7). The fact that a higher proportion of teachers using ADA solutions then go on to implement their ILSs in the classroom (Event_7) also suggests that ADA solutions might alleviate certain hurdles of implementing IBL in authentic settings. Nevertheless, challenges remain in this area (e.g., time, infrastructure, or interoperability constraints), which may not be solvable exclusively through ADA solutions (Event_6).

IBL-specific support Many of the proposed ADA solutions feature IBL-specific concepts (like the inquiry phases), which were well received by teachers (Event_3, Event_4, Event_5). Still, the pedagogical support for designing and enacting effective IBL activities is still in its infancy in Go-Lab. Again, systemic challenges like the lack of teacher training (Blanchard et al., 2009; Hofer \& Lembens, 2019; Silm et al., 2017) and expert feedback (Event_6) make this kind of support a target for future ADA solutions.

These findings paint a promising overall picture of the ADA support for IBL orchestration in Go-Lab. However, future evaluations should look at the validity and reliability of these solutions by both teachers and students (e.g., using the Evaluation Framework for Learning Analytics proposed by Scheffel et al., 2017).

\subsection{Design Guidelines for Solutions That Align Design and Analytics for IBL}

Our interaction with teachers and the different attempts at implementing ADA solutions during the DBR process have unearthed a number of lessons in the design of technologies leveraging learning design and analytics for the orchestration of IBL, which 
can be valuable beyond the Go-Lab community.

1. One need, multiple solutions. Considering the variety of teachers' contextual constraints (from classroom space and furniture to local culture or curriculum) and teaching styles, a single orchestration solution is not likely to work across the board. Thus, as pointed out in previous literature (Gašević et al., 2016), a single orchestration need may require multiple, apparently redundant, solutions. Early user involvement with many different types of teachers is highly recommended to uncover this variety of constraints (Prieto et al., 2019).

2. Time is paramount-develop for time. The most influential factor conditioning the adoption of our ADA solutions was the tension between the limited time, attention, and effort that teachers have and the increased demands that IBL approaches place on their shoulders. As pointed out in learning analytics reviews (Rodríguez-Triana et al., 2017; Schwendimann et al., 2016), the design of ADA solutions should adjust to the timing, cognitive, and interaction demands of each moment. During the learning activity, the emphasis is on regulation and intervention, with little time and attention for processing data. Therefore, solutions should offer inquiry-wide, coarse-grained awareness with a focus on time or deviations from the plan. This information should be glanceable, on both tiny mobile displays and wall projectors (see the previous guideline). In contrast, design-time and post hoc reflection may allow for more detailed information (e.g., about the usage of a single resource, or the paths followed by students throughout the inquiry), which may require more careful interpretation.

3. Blend into the existing ecosystem. It is essential that IBL technologies and ADA solutions "play nice" with tools or hardware that teachers already use for lessons/inquiries. This involves paying special attention to standards and third-party tools to enable interoperability (Dodero et al., 2017). For instance, using XAPI, apps and labs could homogeneously track student activity, so that later on they could be integrated and analyzed. Also, using OpenSocial API and IMS-LTI, embedded tools could be aware of the context where the actions take place (e.g., the inquiry phases, their content, and the users involved). Later on, ADA solutions could create context-aware analyses using such context and activity data.

4. Design for analytics. Apart from designing for data privacy (as required, e.g., by the General Data Protection Regulation, GDPR, in Europe) (Hoel \& Chen, 2016), the collection of meaningful and reliable data requires careful thought about how students interact with the different parts of the IBL ecosystem. For example, frameworks such as the Learning Analytics Learning Gain Design (LALGD) model could contribute to aligning meaningful data capture with pedagogical intentions and their learning outcomes (Blumenstein, 2020).

5. Involve teachers in the loop. Apart from involving teachers in the design and evaluation of ADA solutions, we should enable practitioners to configure them (Rodríguez-Triana et al., 2018). For instance, they should be able to customize contextual parameters like the amount of time to be devoted by students to the different inquiry phases or the kinds of data that can be collected.

6. Design for orchestrating blended learning. We often underestimate the probability of unexpected events interfering with our plans (Kahneman \& Tversky, 1982). Many IBL cases designed in Go-Lab target face-to-face, classroom-bound sessions. However, as happened in several IBL cases reported in our study, this "planning fallacy" often results in learning becoming blended (due to absent students; latecomers; and "open trajectories," which cannot be predicted easily, or when learners need to finish inquiries at home). ADA support should be designed to enable teacher awareness outside the classroom, including notifications or visualizations of the progress of asynchronous inquiries.

7. Scaffolding non-experts. Given that few teachers have experience with scientific inquiry (Blanchard et al., 2009), scaffolding teachers with pedagogical advice is essential to keeping the time, effort, and effectiveness of non-expert teachers at tolerable levels (Dorier \& García, 2013). Such scaffolding should take into account not only the teachers' pedagogical expertise in IBL (be it through analytics-based recommendations during design, guidelines, or contextualized help) but also their data literacy.

8. Account for the evolution of needs. Our long-term study of teacher orchestration needs uncovered a long and evolving list of needs and challenges for IBL adoption. As needs are fulfilled, teachers find new obstacles that require attention. Furthermore, teachers' expertise with IBL and TEL also evolves over time. Hence, it is necessary to perform iterative, holistic evaluations (cf. our use of the orchestration framework to guide the analyses) over longer periods of time in which both the technological support and teachers' own abilities have a chance to evolve.

9. Researching for adoption (meta-guideline). Our four-year DBR process also showed that researching to foster adoption requires not only longer-term efforts but also going beyond asking users for opinions and expectations into observing (in situ or online) their actual everyday use. Also, it requires involving teachers with very different profiles and gathering data in a wide variety of ways (from large-scale log analyses to single-case in-depth interviews and observations).

These nine design guidelines are in many cases aligned with (or particularize) existing design guidelines to support "orchestrable technology" (Prieto et al., 2015) and general good practices in educational technology usability. For instance, guideline \#2 seems rather related to Dillenbourg and colleagues's (2011) advocacy of minimalism, which in turn is somewhat contradicted 
by guideline \#1 (which defends a certain level of redundancy to address multiple classroom realities). Other guidelines are rather about how the research and technology design process should be followed (\#4, \#5, \#8, and \#9). It is worth noting that these IBL orchestration guidelines are quite distinct from other design guidelines for developers in the field of IBL, which focus on how to design TEL to support student inquiry, not teachers' labour (Quintana et al., 2004).

\section{Conclusions and Future Work}

This paper reports on the lessons learned during a four-year DBR study exploring primary and secondary school teachers' IBL orchestration needs in Go-Lab, and how solutions that align learning design and analytics can fulfill such needs. We have clustered the elicited needs into four main orchestration aspects (theory/IBL, pragmatic constraints, design, and enactment). According to our participant teachers, needs related to the enactment of the learning activities appear most frequently and have the highest priority. To address such teacher needs, several ADA solutions were tested. Our findings highlight how these solutions aided the orchestration of IBL in Go-Lab, especially in terms of awareness, assessment, and reflection. During this process, we have also extracted a list of guidelines for technology developers aiming to support IBL orchestration.

The research presented here is not without limitations. The most obvious one is the fact that our DBR and its outcomes (in terms of both empirical findings and design guidelines) are tied to the context in which our evidence was collected, namely the Go-Lab ecosystem, its teacher community, and the specific ADA solutions that we used in our evaluations. This potential lack of generalizability also lies in the fact that, despite being numerous, our informant teachers were not necessarily representative of all teachers attempting to adopt IBL in different cultures or under different curricula. Moreover, the Go-Lab ecosystem already supported many orchestration tasks (related to both design and enactment). Therefore, while eliciting teacher orchestration needs in IBL, we were able to detect only those not supported yet by the ecosystem. Thus, in the future, it would be interesting to cross-check our findings with those from other IBL platforms.

Our exploration of ADA solutions was biased toward the use of learning design to contextualize/enhance the results of learning analytics. The design-time recommendation systems evaluated in our research have only scratched the surface of the complementary approach: to use learning analytics to analyze and eventually improve learning designs. Much of the future work we envision will involve design-oriented ADA solutions and the analysis of their adoption within Go-Lab. We also expect to improve ADA solutions (e.g., with more accurate tracking of timing indicators, or the combination of activityand content-based analytics) in a way that can be useful for assessing the inquiries. To better understand the added value of the ADA solutions and their associated tangible and intangible costs, existing user-centred (Scheffel et al., 2017) and diagnostic (Rodríguez-Triana et al., 2018) approaches could contribute to better adapting the solutions to their intended users. Finally, there is a need to identify validated and pedagogically grounded indicators for IBL that teachers can trust and integrate into their practice, as noted in the area of collaborative learning by Dimitrakopoulou and colleagues (2006).

Despite these limitations, the list of orchestration needs and guidelines outlined above may be useful for other researchers and learning designers interested in supporting technology-enhanced IBL in the challenging environment of primary and secondary school classrooms. Additionally, the detailed account of our methods and approach can serve as an example for others trying to study this complex phenomenon through DBR in the future.

\section{Acknowledgements}

The authors would like to thank the developers, trainers, and teachers involved in this research for their contributions and ideas.

\section{Declaration of Conflicting Interest}

The authors declared no potential conflicts of interest with respect to the research, authorship, and/or publication of this article.

\section{Funding}

The publication of this article received financial support from the European Union in the context of Go-Lab, Next-Lab, GO-GA, and CEITER (FP7 grant agreement no. 317601, and Horizon 2020 Research and Innovation Programme, grant agreement nos. 731685, 781012, and 669074); the European Regional Development Fund and the National Research Agency of the Spanish Ministry of Science, Innovation and Universities (grant agreement no. TIN2017-85179-C3-2-R); the European Regional Development Fund and the National Research Agency of the Spanish Ministry of Science, Innovation and Universities (grant agreement no. PID2020-112584RB-C32); and the European Regional Development Fund and the Regional Government of Castile and Leon (grant agreement no. VA257P18). 


\section{References}

Alavi, H. S., \& Dillenbourg, P. (2012). An ambient awareness tool for supporting supervised collaborative problem solving. IEEE Transactions on Learning Technologies, 5(3), 264-274. https://doi.org/10.1109/TLT.2012.7

Alfieri, L., Brooks, P. J., Aldrich, N. J., \& Tenenbaum, H. R. (2011). Does discovery-based instruction enhance learning? Journal of Educational Psychology, 103(1), 1-18. https://doi.org/10.1037/a0021017

Bakharia, A., Corrin, L., de Barba, P., Kennedy, G., Gašević, D., Mulder, R., Williams, D., Dawson, S., \& Lockyer, L. (2016). A conceptual framework linking learning design with learning analytics. Proceedings of the Sixth International Conference on Learning Analytics and Knowledge (LAK 2016), 25-29 April 2016, Edinburgh, UK, 329-338. https: //doi.org/10.1145/2883851.2883944

Barab, S., \& Squire, K. (2004). Design-based research: Putting a stake in the ground. Journal of the Learning Sciences, 13(1), 1-14. https://doi.org/10.1207/s15327809j1s1301_1

Blake, C., Scanlon, E., Twiner, A., Collins, T., Jones, A., \& Kerawalla, L. (2013). Analysis of learners' fieldtrip talk during a collaborative inquiry task. Scaling up Learning for Sustained Impact, 32-42. https://doi.org/10.1007/978-3-64240814-4_4

Blanchard, M. R., Southerland, S. A., \& Granger, E. M. (2009). No silver bullet for inquiry: Making sense of teacher change following an inquiry-based research experience for teachers. Science Education, 93(2), 322-360. https: //doi.org/10.1002/sce.20298

Blumenstein, M. (2020). Synergies of learning analytics and learning design: A systematic review of student outcomes. Journal of Learning Analytics, 7(3), 13-32. https://doi.org/10.18608/jla.2020.73.3

de Jong, T., Gillet, D., Rodríguez-Triana, M. J., Hovardas, T., Dikke, D., Doran, R., Dziabenko, O., Koslowsky, J., Korventausta, M., Law, E., Pedaste, M., Tasiopoulou, E., Vidal, G., \& Zacharia, Z. C. (2021). Understanding teacher design practices for digital inquiry-based science learning: The case of Go-Lab. Educational Technology Research and Development, 69, 417-444. https://doi.org/10.1007/s11423-020-09904-z

de Jong, T., Linn, M. C., \& Zacharia, Z. C. (2013). Physical and virtual laboratories in science and engineering education. Science, 340(6130), 305-308. https://doi.org/10.1126/science.1230579

Dillenbourg, P., \& Jermann, P. (2010). Technology for classroom orchestration. New Science of Learning (pp. 525-552). Springer. https://doi.org/10.1007/978-1-4419-5716-0_26

Dillenbourg, P., Zufferey, G., Alavi, H., Jermann, P., Do-Lenh, S., Bonnard, Q., Cuendet, S., \& Kaplan, F. (2011). Classroom orchestration: The third circle of usability. In H. Spada, G. Stahl, N. Miyake, \& N. Law (Eds.), Connecting ComputerSupported Collaborative Learning to Policy and Practice: CSCL2011 Conference Proceedings. Volume I-Long Papers 4-8 July 2011, Hong Kong (pp. 510-517). International Society of the Learning Sciences. https://repository. isls.org/handle/1/2491

Dimitrakopoulou, A., Petrou, A., Martinez, A., Marcos, J. A., Kollias, V., Jermann, P., Harrer, A., Dimitriadis, Y., \& Bollen, L. (2006). State of the Art of Interaction Analysis for Metacognitive Support \& Diagnosis (tech. rep. No. D.31.1). Kaleidoscope NoE. https://hal.archives-ouvertes.fr/hal-00190146/

Dobber, M., Zwart, R., Tanis, M., \& van Oers, B. (2017). Literature review: The role of the teacher in inquiry-based education. Educational Research Review, 22, 194-214. https://doi.org/10.1016/j.edurev.2017.09.002

Dodero, J. M., González-Conejero, E. J., Gutiérrez-Herrera, G., Peinado, S., Tocino, J. T., \& Ruiz-Rube, I. (2017). Trade-off between interoperability and data collection performance when designing an architecture for learning analytics. Future Generation Computer Systems, 68, 31-37. https://doi.org/10.1016/j.future.2016.06.040

Dorier, J.-L., \& García, F. J. (2013). Challenges and opportunities for the implementation of inquiry-based learning in day-to-day teaching. ZDM, 45(6), 837-849. https://doi.org/10.1007/s11858-013-0512-8

Emin-Martinez, V., Hansen, C., Rodríguez-Triana, M. J., Wasson, B., Mor, Y., Dascalu, M., Ferguson, R., \& Pernin, J.-P. (2014). Towards teacher-led design inquiry of learning. eLearning Papers, (36). https://infoscience.epfl.ch/record/234556

Friesel, A., Cojocaru, D., \& Avramides, K. (2015). Identifying how PELARS-project can support the development of new curriculum structures in engineering education. Proceedings of the Third Experiment@ International Conference, 2-4 June 2015, Ponta Delgada, Azores, Portugal, 219-223. https://doi.org/10.1109/EXPAT.2015.7463269

Furtak, E. M., Seidel, T., Iverson, H., \& Briggs, D. C. (2012). Experimental and quasi-experimental studies of inquiry-based science teaching: A meta-analysis. Review of Educational Research, 82(3), 300-329. https://doi.org/10.3102/ 0034654312457206

Gašević, D., Dawson, S., Rogers, T., \& Gasevic, D. (2016). Learning analytics should not promote one size fits all: The effects of instructional conditions in predicting academic success. The Internet and Higher Education, 28, 68-84. https://doi.org/10.1016/j.iheduc.2015.10.002 
Gillet, D., de Jong, T., Sotiriou, S., \& Salzmann, C. (2013). Personalised learning spaces and federated online labs for STEM education at school: Supporting teacher communities and inquiry learning. Proceedings of the 15th IEEE Global Engineering Education Conference (EDUCON 2013), 13-15 March 2013, Berlin, Germany, 769-773. https: //doi.org/10.1109/EduCon.2013.6530194

Gluga, R., Kay, J., Lister, R., Simon, Charleston, M., Harland, J., \& Teague, D. (2013). A conceptual model for reflecting on expected learning vs. demonstrated student performance. Proceedings of the 15th Australasian Computing Education Conference (ACE 2013), 29 January-1 February 2013, Adelaide, Australia, 77-86. https://dl.acm.org/doi/10.5555/ 2667199.2667208

Guéraud, V., Lejeune, A., Adam, J.-M., Dubois, M., \& Mandran, N. (2009). Supervising distant simulation-based practical work: Environment and experimentation. In U. Cress, V. Dimitrova, \& M. Specht (Eds.), Learning in the Synergy of Multiple Disciplines (pp. 602-608). Springer. https://doi.org/10.1007/978-3-642-04636-0_55

Harris, C. J., \& Rooks, D. L. (2010). Managing inquiry-based science: Challenges in enacting complex science instruction in elementary and middle school classrooms. Journal of Science Teacher Education, 21(2), 227-240. https://doi.org/10. 1007/s10972-009-9172-5

Hernández-Leo, D., Martinez-Maldonado, R., Pardo, A., Muñoz-Cristóbal, J. A., \& Rodríguez-Triana, M. J. (2018). Analytics for learning design: A layered framework and tools: Analytics layers for learning design. British Journal of Educational Technology, 3(1), 139-152. https://doi.org/10.1111/bjet.12645

Hoel, T., \& Chen, W. (2016). Privacy-driven design of learning analytics applications-Exploring the design space of solutions for data sharing and interoperability. Journal of Learning Analytics, 3(1), 139-158. https://doi.org/10.18608/jla.2016. 31.9

Hofer, E., \& Lembens, A. (2019). Putting inquiry-based learning into practice: How teachers changed their beliefs and attitudes through a professional development program. Chemistry Teacher International, 1(2), 20180030. https: //doi.org/10.1515/cti-2018-0030

Holstein, K., McLaren, B. M., \& Aleven, V. (2019). Designing for complementarity: Teacher and student needs for orchestration support in AI-enhanced classrooms. Proceedings of the 20th International Conference on Artificial Intelligence in Education (AIED 2019), 25-29 June 2019, Chicago, IL, USA, 157-171. https://doi.org/10.1007/978-3-030-232047_14

Hsu, Y.-S., Lai, T.-L., \& Hsu, W.-H. (2015). A design model of distributed scaffolding for inquiry-based learning. Research in Science Education, 45(2), 241-273. https://doi.org/10.1007/s11165-014-9421-2

Jona, K., \& Uttal, D. (2013). Don't forget the teacher: New tools to support broader adoption of remote labs. Proceedings of the 10th International Conference on Remote Engineering and Virtual Instrumentation (REV 2013), 6-8 February 2013, Sydney, Australia. https://doi.org/10.1109/REV.2013.6502910

Kahneman, D., \& Tversky, A. (1982). Intuitive prediction: Biases and corrective procedures. Judgment under Uncertainty: Heuristics and Biases (pp. 414-421). Cambridge University Press. https://doi.org/https://doi.org/10.1017/ CBO9780511809477.031

Kirschner, P. A., Sweller, J., \& Clark, R. E. (2006). Why minimal guidance during instruction does not work: An analysis of the failure of constructivist, discovery, problem-based, experiential, and inquiry-based teaching. Educational Psychologist, 4l(2), 75-86. https://doi.org/10.1207/s15326985ep4102_1

Kotler, P., \& Armstrong, G. (2010). Principles of Marketing. Pearson Education.

Lejeune, A., Ney, M., Weinberger, A., Pedaste, M., Bollen, L., Hovardas, T., Hoppe, U., \& de Jong, T. (2009). Learning activity spaces: Towards flexibility in learning design? Proceedings of the Ninth IEEE International Conference on Advanced Learning Technologies (ICALT 2009), 15-17 July 2009, Riga, Latvia, 433-437. https://doi.org/10.1109/ICALT.2009. 100

Lockyer, L., \& Dawson, S. (2011). Learning designs and learning analytics. Proceedings of the First International Conference on Learning Analytics and Knowledge (LAK 2011), 27 February-1 March 2011, Banff, AB, Canada, 153-156. https://doi.org/10.1145/2090116.2090140

Lockyer, L., Heathcote, E., \& Dawson, S. (2013). Informing pedagogical action: Aligning learning analytics with learning design. American Behavioral Scientist, 57(10), 1439-1459. https://doi.org/10.1177/0002764213479367

Maaß, K., \& Artigue, M. (2013). Implementation of inquiry-based learning in day-to-day teaching: A synthesis. ZDM, 45(6), 779-795. https://doi.org/10.1007/s11858-013-0528-0

Mangaroska, K., \& Giannakos, M. N. (2018). Learning analytics for learning design: A systematic literature review of analytics-driven design to enhance learning. IEEE Transactions on Learning Technologies, 12(4), 516-534. https: //doi.org/10.1109/TLT.2018.2868673

Manske, S., \& Cao, Y. (2015). Specifications of the Learning Analytics, Scaffolding Services, and Add-on Services-Final (tech. rep. No. D4.6). Go-Lab. https://nextlab.golabz.eu/sites/project/files/inline-files/Go-Lab_D4.6-ilovepdf-compressed.pdf 
Manske, S., Hecking, T., Bollen, L., Göhnert, T., Ramos, A., \& Hoppe, H. U. (2014). A flexible framework for the authoring of reusable and portable learning analytics gadgets. Proceedings of the 14th IEEE International Conference on Advanced Learning Technologies (ICALT 14), 7-9 July 2014, Athens, Greece, 254-258. https://doi.org/10.1109/ICALT.2014.80

Mulholland, P., Anastopoulou, S., Collins, T., Feisst, M., Gaved, M., Kerawalla, L., Paxton, M., Scanlon, E., Sharples, M., \& Wright, M. (2012). nQuire: Technological support for personal inquiry learning. IEEE Transactions on Learning Technologies, 5(2), 157-169. https://doi.org/10.1109/TLT.2011.32

Ochoa, X., \& Ternier, S. (2017). Technical learning infrastructure, interoperability and standards. In E. Duval, M. Sharples, \& R. Sutherland (Eds.), Technology Enhanced Learning (pp. 145-155). Springer. https://doi.org/10.1007/978-3-31902600-8_14

Pedaste, M., Lazonder, A., Raes, A., Wajeman, C., Moore, E., \& Girault, I. (2015). Grand challenge problem 3: Empowering science teachers using technology-enhanced scaffolding to improve inquiry learning. SpringerBriefs in Education (pp. 17-20). Springer. https://doi.org/https://doi.org/10.1007/978-3-319-12562-6_4

Pedaste, M., Mäeots, M., Siiman, L. A., de Jong, T., van Riesen, S. A. N., Kamp, E. T., Manoli, C. C., Zacharia, Z. C., \& Tsourlidaki, E. (2015). Phases of inquiry-based learning: Definitions and the inquiry cycle. Educational Research Review, 14, 47-61. https://doi.org/10.1016/j.edurev.2015.02.003

Prieto, L. P. (2015). Classroom technology design guidelines: A focused survey. Proceedings of the Orchestrated Collaborative Classroom Workshop, 7 June 2015, Gothenburg, Sweden, 10-14. http://ceur-ws.org/Vol-1411/paper-03.pdf

Prieto, L. P., Dimitriadis, Y., \& Looi, C.-K. (2015). Orchestration in learning technology research: Evaluation of a conceptual framework. Research in Learning Technology, 23. https://doi.org/10.3402/rlt.v23.28019

Prieto, L. P., Holenko Dlab, M., Gutiérrez, I., Abdulwahed, M., \& Balid, W. (2011). Orchestrating technology enhanced learning: a literature review and a conceptual framework. International Journal of Technology Enhanced Learning, 3(6), 583-598. https://doi.org/10.1504/IJTEL.2011.045449

Prieto, L. P., Rodríguez-Triana, M. J., Martínez-Maldonado, R., Dimitriadis, Y., \& Gašević, D. (2019). Orchestrating Learning Analytics (OrLA): Supporting inter-stakeholder communication about adoption of learning analytics at the classroom level. Australasian Journal of Educational Technology, 35(4). https://doi.org/10.14742/ajet.4314

Quintana, C., Reiser, B. J., Davis, E. A., Krajcik, J., Fretz, E., Duncan, R. G., Kyza, E., Edelson, D., \& Soloway, E. (2004). A scaffolding design framework for software to support science inquiry. Journal of the Learning Sciences, 13(3), 337-386. https://doi.org/10.1207/s15327809jls1303_4

Reimann, P. (2016). Connecting learning analytics with learning research: The role of design-based research. Learning: Research and Practice, 2(2), 130-142. https://doi.org/https://doi.org/10.1080/23735082.2016.1210198

Rienties, B., Toetenel, L., \& Bryan, A. (2015). Scaling up learning design: Impact of learning design activities on LMS behavior and performance. Proceedings of the Fifth International Conference on Learning Analytics and Knowledge (LAK 2015), 16-20 March 2015, Poughkeepsie, NY, USA, 315-319. https://doi.org/10.1145/2723576.2723600

Rodríguez-Triana, M. J., \& Holzer, A. (2016). Release of a Sustainable Version of the Go-Lab Portal and the Associated End-User Solutions (tech. rep. No. D5.7). Go-Lab. https://nextlab.golabz.eu/sites/project/files/inline-files/GoLab_D5.7-ilovepdf-compressed.pdf

Rodríguez-Triana, M. J., Martínez-Monés, A., Asensio-Pérez, J. I., \& Dimitriadis, Y. (2015). Scripting and monitoring meet each other: Aligning learning analytics and learning design to support teachers in orchestrating CSCL situations. British Journal of Educational Technology, 46(2), 330-343. https://doi.org/10.1111/bjet.12198

Rodríguez-Triana, M. J., Prieto, L. P., Ley, T., de Jong, T., \& Gillet, D. (2020). Social practices in teacher knowledge creation and innovation adoption: A large-scale study in an online instructional design community for inquiry learning. International Journal of Computer-Supported Collaborative Learning, 15(4), 445-467. https://doi.org/10.1007/s11412-020-09331-5

Rodríguez-Triana, M. J., Prieto, L. P., Martínez-Monés, A., Asensio-Pérez, J. I., \& Dimitriadis, Y. (2018). The teacher in the loop: Customizing multimodal learning analytics for blended learning. Proceedings of the Eighth International Conference on Learning Analytics and Knowledge (LAK 2018), 5-9 March 2018, Sydney, Australia, 417-426. https://doi.org/10.1145/3170358.3170364

Rodríguez-Triana, M. J., Prieto Santos, L. P., Vozniuk, A., Shirvani Boroujeni, M., Schwendimann, B. A., Holzer, A. C., \& Gillet, D. (2017). Monitoring, awareness and reflection in blended technology enhanced learning: A systematic review. International Journal of Technology Enhanced Learning, 9(2/3), 126-150. https://doi.org/10.1504/IJTEL.2017.084489

Scheffel, M., Drachsler, H., Toisoul, C., Ternier, S., \& Specht, M. (2017). The proof of the pudding: Examining validity and reliability of the evaluation framework for learning analytics. In É. Lavoué, H. Drachsler, K. Verbert, J. Broisin, \& M. Pérez-Sanagustín (Eds.), Data Driven Approaches in Digital Education (pp. 194-208). Springer International Publishing. https://doi.org/10.1007/978-3-319-66610-5_15 
Schwendimann, B. A., Rodríguez-Triana, M. J., Vozniuk, A., Prieto, L. P., Boroujeni, M. S., Holzer, A., Gillet, D., \& Dillenbourg, P. (2016). Perceiving learning at a glance: A systematic literature review of learning dashboard research. IEEE Transactions on Learning Technologies, 10(1), 30-41. https://doi.org/10.1109/TLT.2016.2599522

Sergis, S., Sampson, D. G., Rodríguez-Triana, M. J., Gillet, D., Pelliccione, L., \& de Jong, T. (2019). Using educational data from teaching and learning to inform teachers' reflective educational design in inquiry-based STEM education. Computers in Human Behavior, 92, 724-738. https://doi.org/10.1016/j.chb.2017.12.014

Seymour, E., Hunter, A.-B., Laursen, S. L., \& DeAntoni, T. (2004). Establishing the benefits of research experiences for undergraduates in the sciences: First findings from a three-year study. Science Education, 88(4), 493-534. https: //doi.org/10.1002/sce.10131

Sharples, M. (2013). Shared orchestration within and beyond the classroom. Computers \& Education, 69, 504-506. https: //doi.org/10.1016/j.compedu.2013.04.014

Sharples, M., \& Anastopoulou, S. (2012). Designing orchestration for inquiry learning. In K. Littleton, E. Scanlon, \& M. Sharples (Eds.), Orchestrating Inquiry Learning (pp. 69-85). Routledge. http://www.routledge.com/books/details/ 9780415601139/

Shen, H., Liang, L., Law, N., Hemberg, E., \& O'Reilly, U.-M. (2020). Understanding learner behavior through learning design informed learning analytics. Proceedings of the Seventh ACM Conference on Learning @ Scale (L@ S 2020), 12-20 August 2020, online, 135-145. https://doi.org/10.1145/3386527.3405919

Silm, G., Tiitsaar, K., Pedaste, M., Zacharia, Z. C., \& Papaevripidou, M. (2017). Teachers' readiness to use inquiry-based learning: An investigation of teachers' sense of efficacy and attitudes toward inquiry-based learning. Science Education International, 28(4), 315-325. https://eric.ed.gov/?id=EJ1161535

Slotta, J. D., Tissenbaum, M., \& Lui, M. (2013). Orchestrating of complex inquiry: Three roles for learning analytics in a smart classroom infrastructure. Proceedings of the Third International Conference on Learning Analytics and Knowledge (LAK 2013), 8-12 April 2013, Leuven, Belgium, 270-274. https://doi.org/10.1145/2460296.2460352

Voet, M., \& De Wever, B. (2019). Teachers' adoption of inquiry-based learning activities: The importance of beliefs about education, the self, and the context. Journal of Teacher Education, 70(5), 423-440. https://doi.org/10.1177/ 0022487117751399

Vozniuk, A., Rodríguez-Triana, M. J., Holzer, A., Govaerts, S., Sandoz, D., \& Gillet, D. (2015). Contextual learning analytics apps to create awareness in blended inquiry learning. Proceedings of the 2015 International Conference on Information Technology Based Higher Education and Training (ITHET 2015), 11-13 June 2015, Lisbon, Portugal, 1-5. https: //doi.org/10.1109/ITHET.2015.7218029

Wang, F., \& Hannafin, M. J. (2005). Design-based research and technology-enhanced learning environments. Educational Technology, Research and Development, 53(4), 5-23. https://doi.org/10.1007/BF02504682

Wiley, K. J., Dimitriadis, Y., Bradford, A., \& Linn, M. C. (2020). From theory to action: Developing and evaluating learning analytics for learning design. Proceedings of the 10th International Conference on Learning Analytics and Knowledge (LAK 2020), 23-27 March 2020, Frankfurt, Germany, 569-578. https://doi.org/10.1145/3375462.3375540

Zacharia, Z. C., Manoli, C., Xenofontos, N., De Jong, T., Pedaste, M., van Riesen, S. A., Kamp, E. T., Mäeots, M., Siiman, L., \& Tsourlidaki, E. (2015). Identifying potential types of guidance for supporting student inquiry when using virtual and remote labs in science: A literature review. Educational Technology Research and Development, 63(2), 257-302. https://doi.org/10.1007/s11423-015-9370-0 